Article

\title{
Staff, Symbol and Melody Detection of Medieval Manuscripts Written in Square Notation Using Deep Fully Convolutional Networks
}

\author{
Christoph Wick * (D), Alexander Hartelt (D) and Frank Puppe \\ Chair for Artificial Intelligence and Applied Computer Science, University of Würzburg, \\ 97074 Würzburg, Germany \\ * Correspondence: christoph.wick@informatik.uni-wuerzburg.de
}

Received: 16 May 2019; Accepted: 22 June 2019; Published: 29 June 2019

check for updates

\begin{abstract}
Even today, the automatic digitisation of scanned documents in general, but especially the automatic optical music recognition (OMR) of historical manuscripts, still remains an enormous challenge, since both handwritten musical symbols and text have to be identified. This paper focuses on the Medieval so-called square notation developed in the 11th-12th century, which is already composed of staff lines, staves, clefs, accidentals, and neumes that are roughly spoken connected single notes. The aim is to develop an algorithm that captures both the neumes, and in particular its melody, which can be used to reconstruct the original writing. Our pipeline is similar to the standard OMR approach and comprises a novel staff line and symbol detection algorithm based on deep Fully Convolutional Networks (FCN), which perform pixel-based predictions for either staff lines or symbols and their respective types. Then, the staff line detection combines the extracted lines to staves and yields an $F_{1}$-score of over $99 \%$ for both detecting lines and complete staves. For the music symbol detection, we choose a novel approach that skips the step to identify neumes and instead directly predicts note components (NCs) and their respective affiliation to a neume. Furthermore, the algorithm detects clefs and accidentals. Our algorithm predicts the symbol sequence of a staff with a diplomatic symbol accuracy rate (dSAR) of about $87 \%$, which includes symbol type and location. If only the NCs without their respective connection to a neume, all clefs and accidentals are of interest, the algorithm reaches an harmonic symbol accuracy rate (hSAR) of approximately 90\%. In general, the algorithm recognises a symbol in the manuscript with an $F_{1}$-score of over $96 \%$.
\end{abstract}

Keywords: optical music recognition; historical document analysis; medieval manuscripts; neume notation; fully convolutional neural networks

\section{Introduction}

The digitisation and encoding of historical music manuscripts is an ongoing area of research for many scientists. The aim is to preserve the vast amount of cultural heritage but also to provide musical information in a machine-readable form (e.g., ${ }^{* *}$ kern (http:/ / www.humdrum.org/rep/kern/), MEI (https:/ / music-encoding.org/), or MusicXML [1]). For one thing, this enables music scientists to apply large-scale musical analysis such as detecting similarities of melodies, creating musical grammars, or comparing different versions of the same piece of music. Furthermore, the digital transcriptions are published in a collection [2] that is searchable and provides tools for data retrieving. The current Corpus Monodicum project at the University of Würzburg is dedicated to the exploration and edition of historically significant music, editorially untapped stocks of unanimous ecclesiastical and secular music of the European Middle Ages with Latin text. Two volumes [3,4] have already been published; however, the majority of material of interest has not been converted into machine actionable form yet. Our proposed Optical Music Recognition (OMR) aims to speed up this process considerably. 
If in the process of encoding, the data maintains positional information of symbols or regions relative to original manuscript, ambiguities, corrections or other sources of manual interpretation can be looked up and verified in the original document. In general, this should highly improve the quality of research and reproducibility.

Currently, the digital acquisition heavily relies on human effort because the ancient manuscripts particularly suffer from degradation and non-standardised fonts, glyphs or layouts. Therefore novel techniques in the area of artificial intelligence are required to capture the encoded data in a computer readable form. This paper focuses on the digitisation of medieval monophonic music written in square notation, an ancient notation which was developed and used from the 11th-12th century onwards [5]. Compared to even earlier forms, this writing of music is already similar to Common Western Music Notation in the sense that it uses four staff lines, clefs, accidentals and more or less discrete notes that are drawn as squares (compare Figure 1). However, unlike modern notation, notes are mostly connected to groups, the so-called neumes witch depict a small segment of melodic motion.

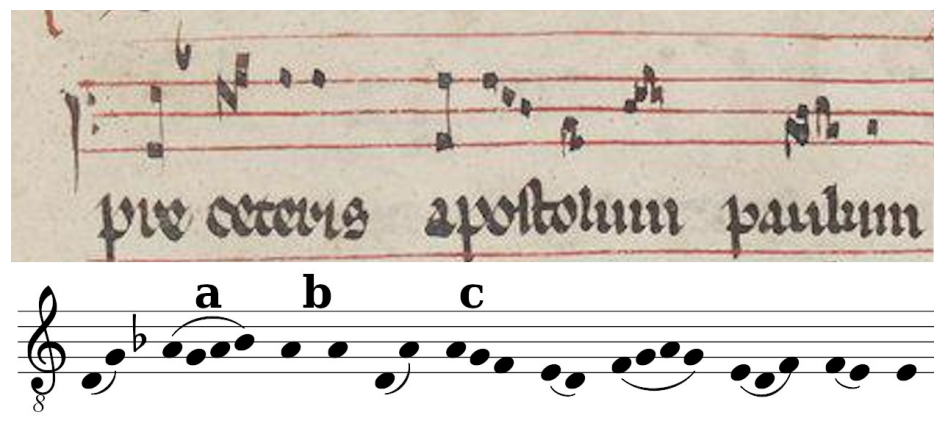

Figure 1. An example transcription equivalent in modern notation (bottom image) based on a neume notation (upper image). A neume consisting of graphical connected note components (NCs) (a) is visualised by a slur, each new neume starts with an increased space (b), logical connected NCs are notated with small note space (c). The modern notation maintains the relevant information for performing the historical neume notation. The example image is taken from our corpus introduced in Section 3.

Historically, neumes arise from single solid or broken stroke drawings that visually followed the pitch levels. Figure 1 also shows an actual transcription of the upper line taken from our data set in a modernised form where each note component (NC) is a single note. The graphical connectivity of two NCs, which is in most cases straightforward to decide, is marked by a slur (a). Example (b) shows two notes that are two single tone neumes and are therefore notated with a larger space. Logical connected NCs are visualised by a smaller space between two notes (c). This, however, is in some cases difficult to decide because there is no difference between a neume consisting of multiple single NCs or multiple neumes comprising one NC. In this example, the syllables each belonging to different neumes dissolve this ambiguity.

The aim of this paper is, on the one hand, to detect the staff lines that form staves and on the other hand, to locate all musical symbols including the discrete note position of the neumes, so that the musical relevant information including the positions in the original document is fully recognised and preserved. Both tasks are conducted separately by usage of two Fully Convolutional Networks (FCNs), which predict pixel based locations of staff lines or music symbols, respectively. Further post-processing steps extract the actual positions and symbol types.

In contrast to the works of other researchers $[6,7]$ that first detect and classify neumes and then resolve them into single NCs to gain the distinct pitches, our algorithm combines this step by directly yielding the described transcription equivalent. However, the actual neumes in the original manuscript can be reconstructed and thus no information is lost. The advantage of our approach is that we bypass the ambiguities of neumes that are very similar to each other but yield the same pitches. Furthermore, no subsequent faults can arise. Eventually, we conduct an evaluation that both ignores and includes 
the neume connectivity to measure either only the quality of the detected melody or the capability to digitise the complete square notation.

To summarise, the main contributions of this work regarding an OMR of manuscripts written in square notation are the following:

- Development of a very robust staff line detection algorithm based on FCNs, which identifies almost all staves and their related staff lines.

- Proposal of a symbol detection algorithm which applies an FCN to the detected staves. The algorithm locates clefs, accidentals and notes with their respective affiliation to neumes. Thereby, it is possible to fully capture and reconstruct the melodic sequence.

- Evaluations of both new algorithms on 49 pages comprising 510 staves and 16,731 annotated symbols. We consider, among other things, the effect of only a few training examples and the generalisation to new manuscripts.

The remainder of this paper is structured as follows: First, related work is listed and discussed. Afterwards, the algorithms for staff and symbol detection are described and we evaluate the algorithms independently, including the experiments that measure the amount of required training data. We conclude this paper by giving an outlook on our future work.

\section{Related Work}

The digitisation on manuscripts is an ongoing area of music research but also for textual documents. The special focus of this paper is monophonic music, where the music forms a temporal sequence and hence it is sufficient to only detect one symbol at a point in time. In the following, we list work related to OMR on monophonic music detection and to music detection on historical documents in general. With the recent overwhelming success of deep learning, CNNs acting on raw input data instead of preprocessed images (e.g., staff line removal) became very popular and yielded state-of-the-art performance. Since OMR is basically a sequence to sequence task, recurrent networks such as LSTMs are also promising to use.

\subsection{OMR on Contemporary Notation}

Baró-Mas et al. [8] use (bidirectional) LSTM networks to predict the pitch and rhythm of notes and other musical symbols for example rests and clefs in images, each containing a single line of monophonic music. The outputs of the network are two sequences. The first one aims to predict the pitch of notes or the type of other symbols like clefs (54 classes in total), while the second one indicates the rhythm of notes or a no note label (26 classes). For training the network, they implement two different loss functions that consider both target sequences. One computes the weighted euclidean loss, the other implements a multi-label soft margin loss. To evaluate their algorithm, two different data sets are used which both consist of monophonic music in modern notation, however one is printed and one handwritten. Experiments on the first dataset which comprises incipts from the RISM data set [9] yield a symbol/pitch error rate of $1.5 \%$ and a rhythm error rate of $2.0 \%$. The total error for both properties to be correct is $2.8 \%$. As a second data set, they use one page of the CVC-MUSICMA dataset [10]. After manually labelling six staves, they extend the number of different staves by varying the order of bars within a single staff. During training, data augmentation is used and all lines from the printed data set were added. The error of the symbol/pitch and rhythm detection is $47 \%$ and $43 \%$, respectively, yielding a total error rate of $65 \%$.

A similar attempt to learn music recognition on modern monophonic printed music was proposed by van der Wel and Ullrich [11]. However, compared to Reference [8], they use preliminary CNN layers and an encoder/decoder structure for their neural network. The CNN/LSTM based encoder maps a line image into one fixed size state representation by processing the line sequentially. The decoder consecutively decodes this state and predicts a sequence of pitches, duration, and finally a stop mark. This overall procedure is based on, and very similar to, the sequence to sequence task used 
for machine translation [12]. In total there are 108 pitch and 48 duration categories in their data set, which is compiled from MusicXML [1] scores from the MuseScore (https://musescore.org/) sheet music archive. In contrast to the normalised edit-distance, a rather strict metric is used that cannot handle insertions or deletions. They align the prediction and ground truth (GT) sequence label by label and count the number of correct predictions. Thus, if a label is deleted or inserted all subsequent notes are usually false. Their final model which is trained with data augmentation, yields a pitch and duration accuracy of $81 \%$, and $94 \%$, respectively. The total accuracy of notes is $80 \%$.

A third very promising attempt to predict monophonic music was made by Calvo-Zaragoza and Rizo [13]. They use CNN/LSTM hybrids combined with a CTC-loss-function [14] as model architecture, a technique that already succeeded for handwritten and printed optical character recognition (OCR) $[15,16]$, or speech recognition $[17,18]$, which are both sequence to sequence tasks. The advantage of this loss function is that it does not require position accurate labelling in the GT. Only the target sequence and input data are obligatory, out of which the network automatically learns the alignment. The drawback of this method is that each combination of pitch and rhythm semantically requires a distinct label. Moreover, a key-signature can, for instance, either be a single symbol or be dissolved in individual accidentals. The first so-called semantic representation requires 1781 classes in total, while the second agnostic representation only uses 758 classes. To perform experiments they created the so-called PrIMuS (available at https://grfia.dlsi.ua.es/primus/) (Printed Images of Music Staves) data set containing 87,678 real-music incipts which are rendered by Verivio [19], a web-based music engraver. The evaluation yields a sequence error rate of $17.9 \%$ and $12.5 \%$ in the agnostic and semantic representation, respectively, which is explainable by the higher number of classes. However, the individual symbol error rate is approximately $1 \%$ in both representations.

Compared to the upper work, handwritten music recognition can also be regarded as an object detection task. This approach was proposed by Pacha et al. [20]. Their pipeline uses existing state-of-the-art deep neural networks for object detection such as Faster R-CNN [21] or R-FCN [22] with custom pre-processing and training. A cropped image of a single staff without staff line removal serves as input. They evaluated the MUSCIMA++ dataset [23] which contains over 90,000 symbol annotations of handwritten music distributed in 71 classes. The object detection achieved a mean average precision of over $80 \%$.

\subsection{OMR on Historical Notations}

In the area of historical OMR, Calvo-Zaragoza et al. [24,25] applied Hidden Markov Models (HMM) and an n-gram language model on line images written in mensural notation of the 17th century. This notation is comparable to modern notation, since it is already ruled by very similar symbols. Their handwritten corpus was comprised of 576 staves with 13,863 individual symbols representing for instance notes, rests, or clefs. In total there were 16 different symbol shapes which are located on discrete locations relative to the staff lines. The combination of the position and shape yielded around 200 different classes. As metrics they measured the glyph error rate (GER, symbol shape only) and height error rate (HER, position relative to the staff lines) separately but also computed the combined symbol error rate (SER, shape and position). Their best model reached a GER of $35.2 \%$, a HER of $28.2 \%$, and a SER of $40.4 \%$.

Ramirez and Ohya [7] did notable work on the automatic recognition of handwritten square notation, which is also the focus of our paper. They built a dataset based on 136 pages from the Digital Scriptorium repository (http:/ / www.digital-scriptorium.org/) comprising 847 staves and over 5000 neumes. The greyscale images of the 14th century are a huge challenge, as they suffer from physical degradation, variability in notation styles or non-standardised scan conditions. Their first task detected and extracted staves. They used a brute-force algorithm that matches the original image with a staff template built up from four straight staff lines by varying line and staff distance and orientation. The found optima indicated locations for staves, which were then extracted. The advantage of this method is that individual staff lines need not to be detected; however, the handwritten staff lines must be 
equally distant and almost straight. In total, 802 of all 847 staves were correctly detected ( $95 \%$ recall). In a second task, they performed a symbol detection and classification algorithm. A pattern matching algorithm fed with several different templates for each neume first marked possible symbol locations and found approximately $88 \%$ of all symbols. Then, a SVM classified the symbols into different neume types with an accuracy no lower than $92 \%$ across all classes. Compared to our approach, they did not resolve the individual neumes into single NCs nor detected clefs. But likewise, accidentals were ignored or did not occur.

Vigliensoni et al. [6] focused on pitch detection in their work; however, on documents of the Liber Usualis (a digital version is available at https:/ / archive.org/details/TheLiberUsualis1961) printed in 1961. Using the staff line detection of Miyao [26], the staff line removal of Roach and Tatem (compare e.g. Reference [27]), and an automatic neume classification algorithm trained on 40 pages, they evaluated pitch detection on 20 pages consisting of 2219 neumes and 3114 pitches. The pitch of the first component of a neume was correctly detected for $97 \%$ of all neumes, while only $95 \%$ of all note components including single-tone neumes were found.

In Reference [28], Calvo-Zaragoza et al. propose an approach taken from historical document analysis [29] in the area of OMR. A deep CNN was trained to segment scans of two manuscripts of the 14th and 16th century pixel-wise by assigning each pixel a class selected from background, text, staff line, or symbol. Approximately $80 \%$ of the pixels were background, $10 \%$ were text, $6 \%$ were staff lines and $4 \%$ were symbols. The results showed that, especially at the margin of changing label types, the classification was incorrect, which however should only have a minor impact on the proceeding steps. Furthermore, only a small number of GT instances must be manually created to obtain good results. The evaluation measured the correct label of pixels, particularly those located at the edge of different symbols and yields an average $F_{1}$-score of around $90 \%$. In general, this algorithm which predicts a pixel-wise segmentation only solves one step in an OMR pipeline. The segmentation can be used as an input to feed various classifiers which are, for instance, trained to encode staff lines, staves, symbols or text, to finally output the musical information.

\section{Dataset}

As a dataset we used 49 pages of the manuscript "Graduel de Nevers" (accessible at the Bibliothèque nationale de France (https:/ / gallica.bnf.fr/ark:/12148/btv1b8432301z)) published in the 12th Century. The handwritten music comprises different neume notation styles, only a part is written in square notation, which are folios 2-9 and 246-263. These pages were extracted and further split into three parts that share a similar layout or difficulty based on our human intuition (compare Figure 2).

The first part contained pages with the best available notation quality with fading bleeding. Its staff lines are mostly straight, possibly due to usage of a ruler, and all neumes were very clear and distinct. The second part suffered from bleeding staff lines and was written very narrowly, yielding the most difficult notation. The third part comprised to some extent very unclear neumes and very wavy staff lines. In our experiments, we used these parts to estimate how well our trained algorithms generalise onto unseen layouts by not using all parts for training.

The GT was manually annotated under the supervision of music scientists. For each stave, we stored four staff lines each as a polyline whose coordinates were relative to the the image. The exact start and end of a line was ambiguous due to occasional severe degradation. We further defined the symbols clef, accidental, and neume as part of a staff. A clef consists of a type (C or F), its centre as absolute position on the image and its location relative to the staff lines, which also marks the denoted staff line. In the example line in Figure 1 the F-clef is located on the second line. Since all occurring accidentals are a flat $B$, it is sufficient to store their absolute centre position and their staff line location. Each neume is comprised of single NCs. For each NC, we took its absolute position, its location on the staff lines, and whether it is graphically connected to the previous NC. For example the first neume in Figure 1 consists of two NCs. The first one is located on the first staff line while the second one is graphically connected and is positioned in the second space. The neume (c) in the example line 
comprises three NCs of which none is graphically connected; however, since they belong to the same neume, their logical connection can be derived. Note that, in general, the symbol location in a staff line can be computed using the positions of the staff lines and the global position of the symbol, however there exist many ambiguities as to whether a NC is actually located on a line or in a space. For instance, the first NC of the second last neume in Figure 1 might also be an G instead of an F. In this work, we did not distinguish additional note types such as liquecents or oriscus. Furthermore, unlike the approach in Reference [28], we did not label the images on a pixel-basis, since detecting the actual shape and extent of neumes, clefs, or accidentals is out of the focus of this paper. We were solely interested in transcribing historical neume notations in a digitised form that preserved all melodic information, which is the desired output in almost all cases.

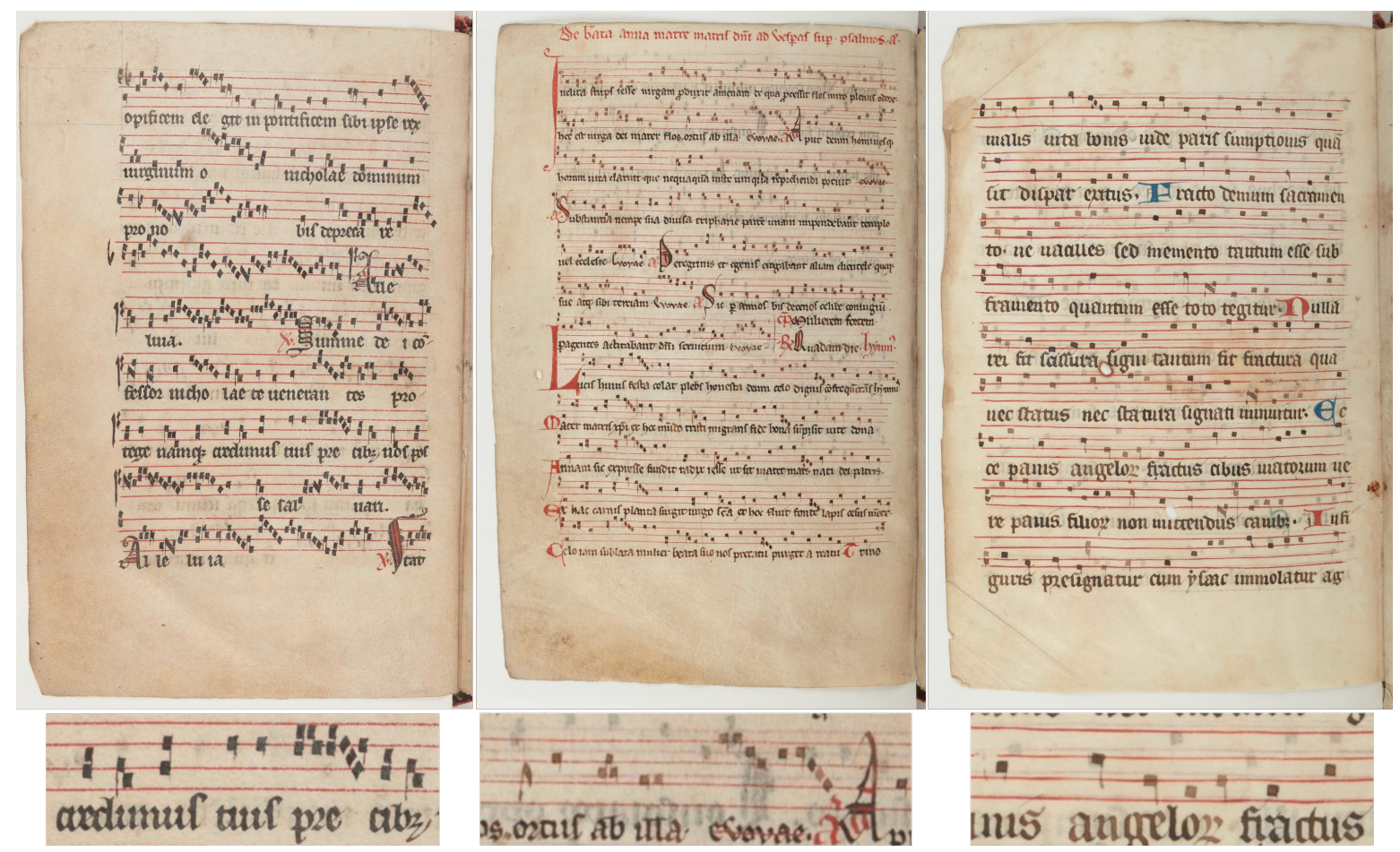

Figure 2. Each page represents parts 1, 2, and 3, respectively. The bottom images provide a zoomed view on the upper pages. While the first page is very clean, both other pages suffer from bleeding and fainter writing. The staff lines in the first and second part are very straight most certainly due to usage of a ruler, whereas the staff lines of the third part are freehand drawn.

Using the described storage scheme, all required information for training and evaluation is preset or can be computed. Table 1 gives an overview of each part's properties by listing its number of pages, the number of staves on these pages, each consisting of four staff lines, and the symbol counts. In total, we annotated more than 16,000 symbols located in more than 500 staves. Compared to the number of symbols, the 65 flat accidentals occur very rarely, which makes the learning of their detection very challenging. Approximately $4 \%$ of the symbols are clefs, yet at least up to 600 clefs can be used as training examples. 
Table 1. Overview of the data set statistics. The data set is split manually into three groups that share similarities in layout or handwriting.

\begin{tabular}{cccccccc}
\hline Part & Pages & Staves & S.-Lines & Symbols & Notes & Clefs & Accid. \\
\hline 1 & 14 & 125 & 500 & 3911 & 3733 & 153 & 25 \\
2 & 27 & 313 & 1252 & 10,794 & 10,394 & 361 & 39 \\
3 & 8 & 72 & 288 & 1666 & 1581 & 84 & 1 \\
\hline Total & 49 & 510 & 2040 & 16,371 & 15,708 & 598 & 65 \\
\hline
\end{tabular}

\section{Methodology}

This section describes the general workflow of the staff line and symbol detection. Furthermore, we provide an introduction to FCNs which are heavily used by the algorithms.

\subsection{Workflow}

The typical OMR pipeline usually breaks down in the following four stages as shown in [30]:

1. image pre-processing,

2. staff line detection and recognition of musical symbols,

3. reconstruction of the musical information, and

4. construction of a musical notation model.

Since the focus of this paper is to output an encoding of staves consisting of staff lines and music symbols that fully captures the written neume notation of the original manuscripts, only the first three steps are included in the proposed workflow. The omitted reconstruction of the output into a modern notation is, however, straightforward (compare Figure 1). Therefore, our pipeline starts with a scanned color image and ends with the reconstructed symbols that are recognised and positioned in a detected staff as seen in Figure 3.

During the pre-processing, first, the raw image was deskewed and converted into grayscale by using OCRopus (https://github.com/tmbdev/ocropy/blob/master/ocropus-nlbin). The deskewing algorithm was initially designed for processing textual documents in an OCR-pipeline, however it generalises flawlessly on our musical data. The algorithm first applied an array of small rotations on the image. Afterwards, for each rotated image all rows were averaged pixel-wise and the variance across the resulting vector was computed, which yielded a score for each initial rotation angle. Finally, finding the maximum yielded the best rotation angle.

Next, our staff line detection algorithm was applied to the pre-processed image. The resulting staff lines were represented as polylines and grouped into single staves. Each staff was then cut out of the pre-processed image and the symbol detection was applied, which yielded a list of symbols including their absolute pixel position. Finally, this pixel position was converted to a location relative to the recognised staff lines to produce the final output. The last step for an actual transcription was a straightforward mapping which decoded these positions based on the detected clefs to actual note names, that is, the pitch. This step can induce subsequent faults if the clef was misclassified. The melody of a line however, which is defined by the intervals that are relative to the NCs, is independent of the clef which only defines the global pitch.

Both the staff line and symbol detection make use of FCNs, which we will introduce in the following. Afterwards, the individual algorithms are described in greater detail. 


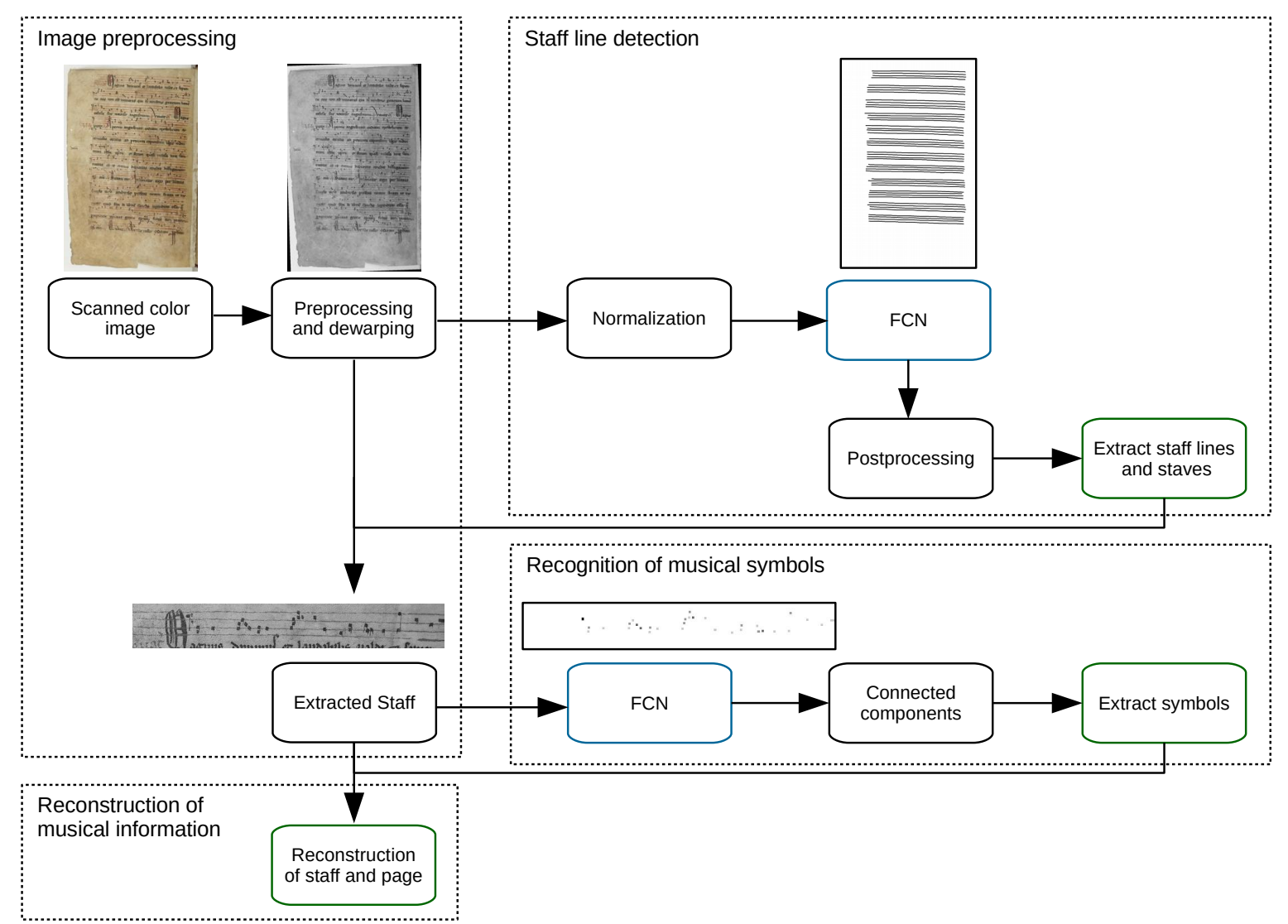

Figure 3. Schematic view of the workflow of the staff line and symbol detection. The small images show the original image and the input and output of the respective Fully Convolutional Network (FCN).

\subsection{FCN Architecture}

FCNs are a novel method for classifying an input image with variable size $(H \times W)$ pixel-wise by assigning a target class to each pixel in the input image. Especially the U-Net structure of [31] poses a new state-of-the-art in several areas.

Our network architecture, which is shown in Figure 4, is a bit simpler than the original U-Net since it has a reduced number of filters in the Convolution Layers and only down scales to a factor of 8 .

In general, the U-Net comprises an encoder/decoder structure that down- and up-scales the input image and thereby applies convolution and pooling operations. Skip-connections directly link the outputs of each scale level of the encoder to the outputs of the decoder by concatenation. Thus, the next higher layer of the decoder knows both the widespread information of the deeper levels and the more fine-granular features of the higher levels of the encoder. The output of the network is a probability distribution over all allowed labels for each input pixel. 


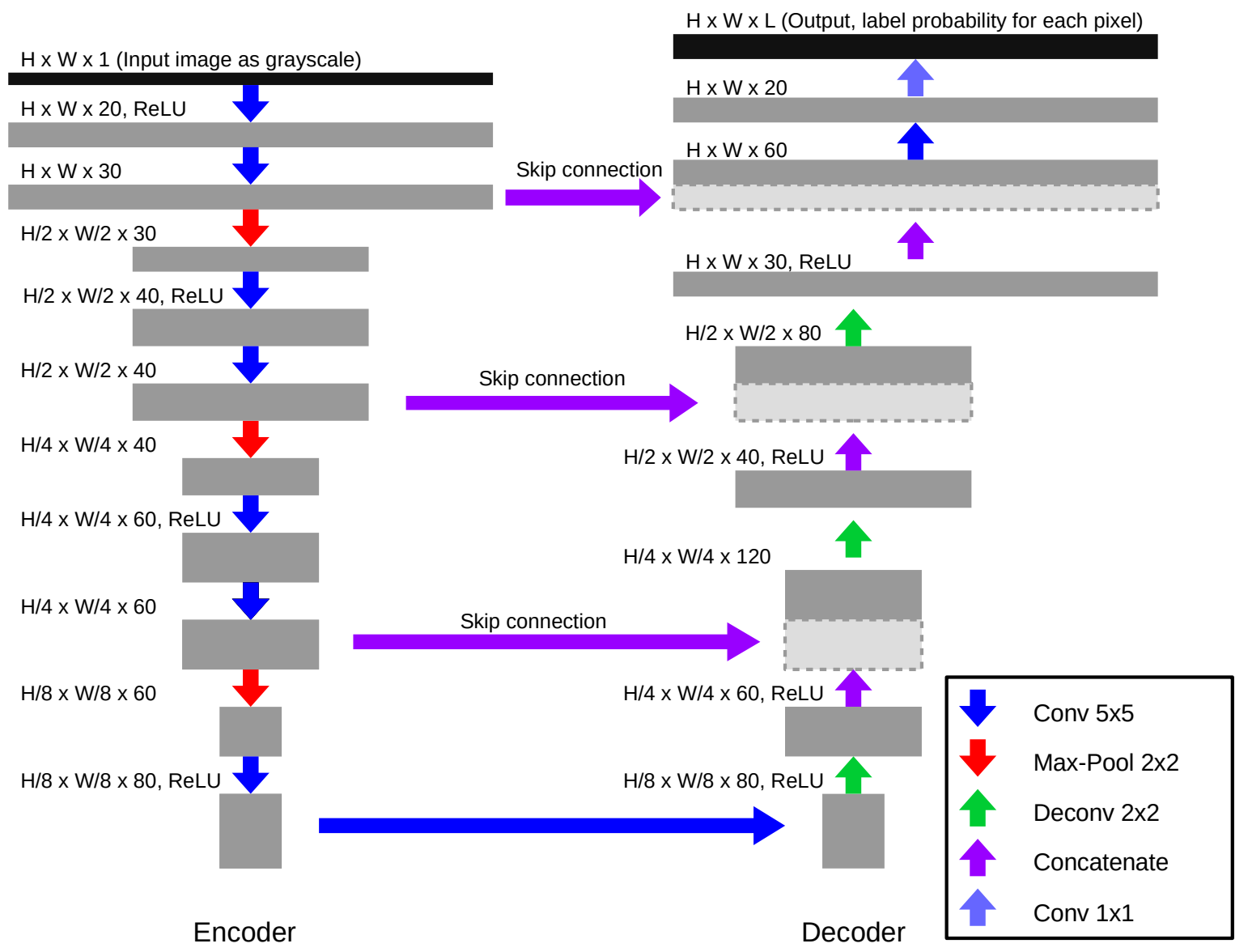

Figure 4. FCN network architecture used for the staff line detection and symbol detection. Both the type and the dimension of each layer is shown.

The network is trained by using a pair of input (e.g., greyscale) image $I$ and a corresponding target label matrix $T$ as GT (compare Figure 5 for example input pairs). The neural network tries to predict the desired label with a probability of one, while all other classes shall be zero. The corresponding loss function is the negative-log-likelihood $L$ which treats each individual pixel prediction independently:

$$
L=-\sum_{x, y} \log P(T \mid I)_{x, y}
$$

where the sum runs over all pixel positions $x, y$ and the matrix $P(T \mid I)$ denotes the probability of the GT labels $T$ given the input $I$. 


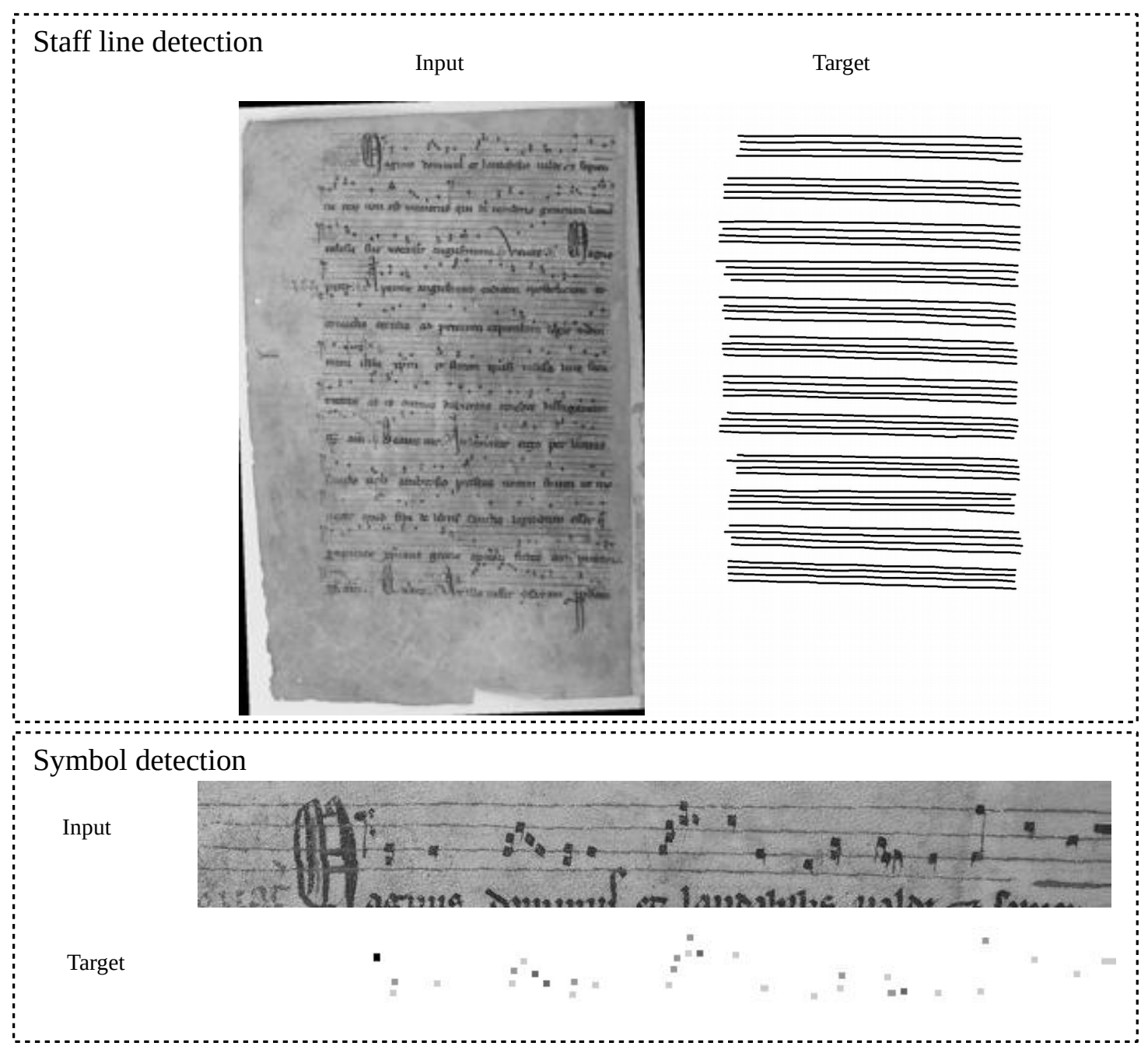

Figure 5. GT pairs of input and target for training the FCN. The upper box shows an example for the staff line detection where the FCN acts on the full page. The staff lines in the GT are drawn as black lines in the target binary image. The symbol detection, instead, expects an image of a single line as input. The different grey levels of the target represent the symbol classes, for example the darkest level indicates a F-clef, while the brightest level marks a NC which is the start of a neume.

\subsection{Staff Line Detection}

Our staff line detection algorithm aims to extract staves and their respective staff lines as a group of polylines from an input image. Each polyline is a list of points located in the original image.

Our algorithm (compare Figure 3) for the staff line detection acts on the deskewed greyscale image of a full page as input and expects as parameters the number of staff lines per staves (four in the case of this paper) and the average staff line distance $d_{\mathrm{SL}}$ which is however approximated by the algorithm of $[32,33] . d_{\mathrm{SL}}$ is used to normalise the original input to an image where $d_{\mathrm{SL}}$ is a fixed value of 10px. The automatic staff line distance approximation algorithm of [33] acts on the binarised version of the input image which is computed by the OCRopus binarisation tool. The idea of the algorithm is based on run-length encoding (RLE) for each column of the binary image. RLE encodes an array of binaray black and white pixels in sequence length, for example, the sequence $\{1110011111000111010\}$ is encoded as $3,2,5,3,3,1,1,1$. The most common black runs represent the height of a single staff line, while the most common white runs denote the staff space, 1 and 3 in the upper example, respectively. We adapted this algorithm by constraining the staff space to be at least twice the staff line height to gain more robust results with noisy data. The actual $d_{\mathrm{SL}}$ is finally approximated by the sum of the staff line height and space. A preliminary experiment shows that on all available pages this algorithm 
correctly approximates $d_{\mathrm{SL}}$, hence all images can be normalised automatically. This normalisation step is only required for the staff line detection but not for the later symbol detection.

The pre-processed and normalised image (a) is then fed into the FCN and postprocessing pipeline (compare Figure 6). The FCN, which is trained to discriminate between staff line and background pixels, predicts a probability map (b)-compare also Figure 5 . A threshold of $50 \%$ corresponding to the class with the highest probability classifies each pixel (c). To prune artefacts, we then apply a horizontal RLE to drop row-wise pixel groups with a length shorter than $6 \mathrm{px}$ (d). The remaining connected components (e) are then used to determine preliminary polylines by computing the vertical centre at each horizontal position for each component (f). Since the staff lines are sometimes interrupted, we apply a postprocessing step that combines polylines which are on a similar height level (g). Finally, we cluster all staff lines into staves by first computing the most common distance of staff lines $\bar{d}_{\mathrm{SL}}$ and then group staff lines with a distance of smaller than $1.5 \cdot \bar{d}_{\mathrm{SL}}$ (h). We only keep staves with four lines, by what staves that might be wrongly classified noise (e.g., bleeding), text or the page margin, are dropped. The remaining grouped polylines represent the final result of the staff line detection algorithm.

(a) Input

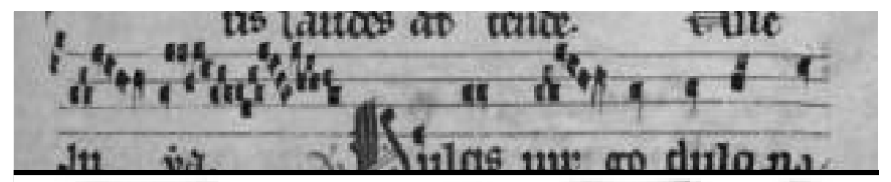

(b) Output (Probability)

(c) Threshold (50\%)

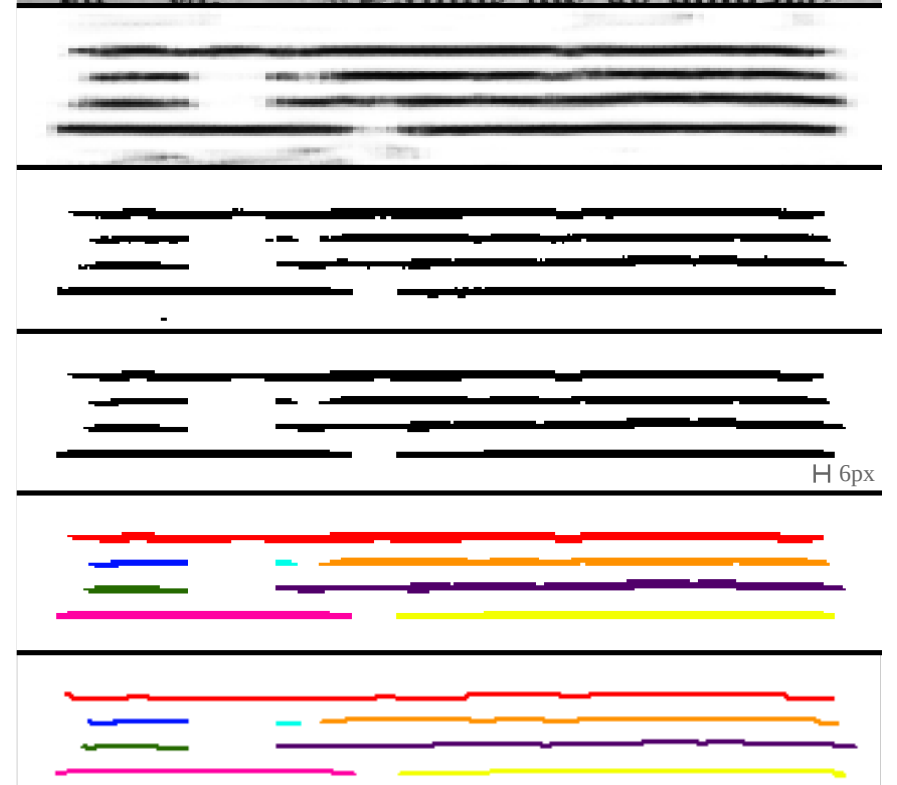

(d) Horizontal runs (Pruning)

(e) Connected Components

(f) Polylines (center of Ccs)

(g) Combine line sections

(h) Group into staves

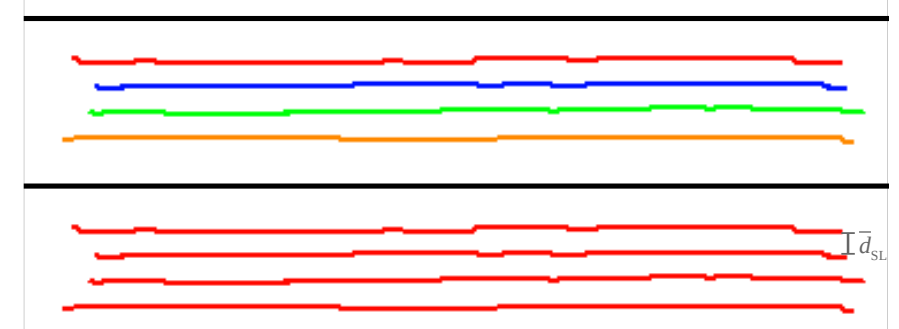

Figure 6. Staff line detection steps including the application of the FCN and the postprocessing. The example images show the output of the respective step. In step $(\mathbf{f}-\mathbf{h})$ the resulting polylines indicating staff lines are drawn, while in the other steps (a-e) the output is a matrix. The run-length encoding (RLE) scale of 6 px relative to the output and the dimension of $\bar{d}_{\mathrm{SL}}$ is shown as reference in gray.

The FCN is trained on GT that solely contains the human annotated staff lines drawn with a thickness of 3 px. During training, we optionally augment the data by randomly rotating the image up to $2^{\circ}$, flipping the image vertically or horizontally, and varying the contrast and brightness up to a 
factor of $2^{[-0.8,0.8]}$ and value of $8 \%$, respectively. Furthermore, the data is scaled with a factor of up to $2^{[-0.1,0.1]}$ on each axis independently.

\subsection{Symbol and Note Component Detection}

The symbol detection algorithm acts on the image of a single staff (see Figure 3). To extract these images, we determine the bounding boxes of each known staff which are extended to the top and bottom by adding $d_{\mathrm{SL}}$, which is computed using the given staff lines. This line image is finally scaled to a fixed height $h_{\text {staff, }}$ which we choose as $80 \mathrm{px}$ (see Section 5.3.2), so that the resolution of the input data is normalised. An example line is shown in the first row of Figure 7.

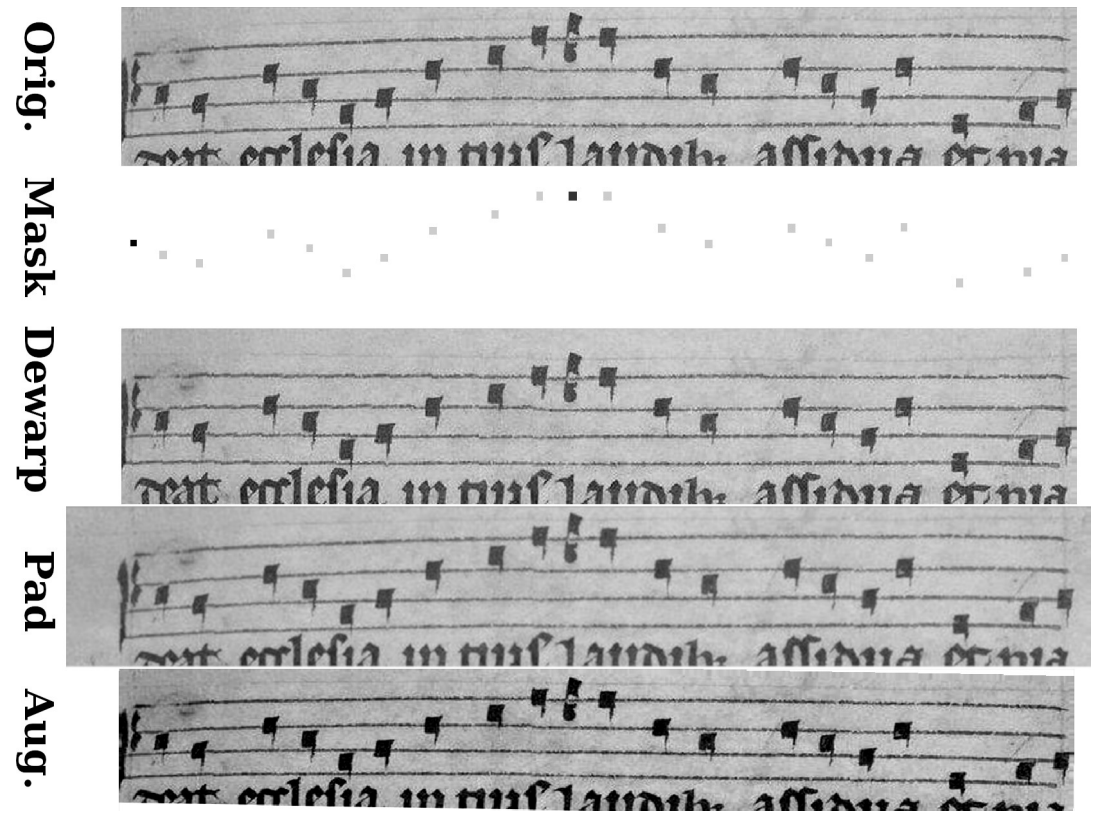

Figure 7. The first line shows the cut off original input image. The next one is the target mask for the FCN in which notes and clefs are marked. The next three lines show the dewarped, padded and an augmented version of the original image.

Similar to the staff line detection, the symbol detector employs the proposed FCN-architecture to classify the input image pixel-wise into seven different classes (see Figure 8): clef-c, clef-f, accidental-flat, initial NC (start of a neume), logical connected NC, graphical connected NC and background. The GT mask for each line is generated by drawing a small box of the respective label at the centre of each symbol (compare Figure 5 and the second image in Figure 7). When training the FCN it aims to predict the correct labels pixel wise, which is why actual symbols and their position and types must be extracted in a proceeding step. For this purpose, we first assign the most probable class to each pixel to receive a label map. Afterwards, connected components are detected on a binary image that either denotes background or any symbol. Each component yields a single symbol whereby the centre of the component equates the symbol position and the type defined by majority voting on the label map. 


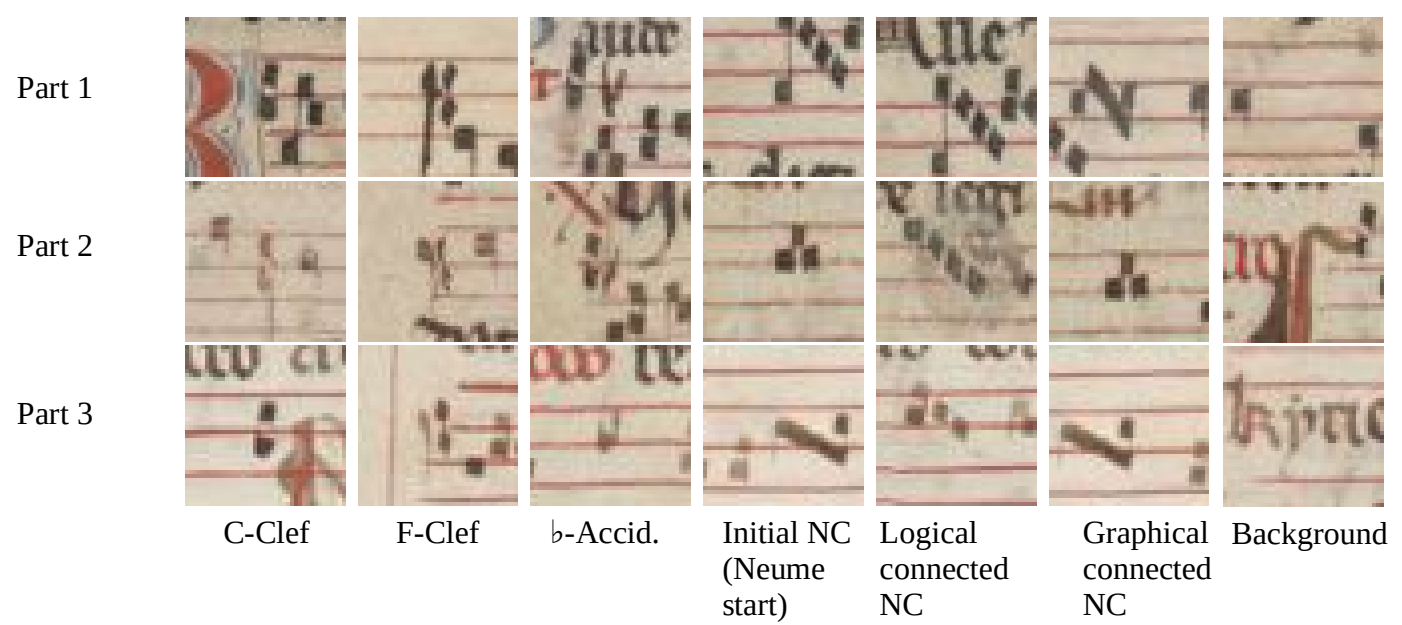

Figure 8. Each column shows cropped instances of the seven classes to be recognised by the symbol detection, respectively for each data set part. The central pixel position defines the type.

Finally, the relative note position is computed based on the detected staff lines (see left image of Figure 9), whereby the relative distance of the $y$-coordinate of each symbol in the staff determines the closest space or staff line. It shows that notes are often "on the line" even though it is visually closer to a space which is why spaces and lines are not distributed equidistant, instead the ratio is $2 / 3$ (see the left zoomed sub figure and for an example the right image of Figure 9).
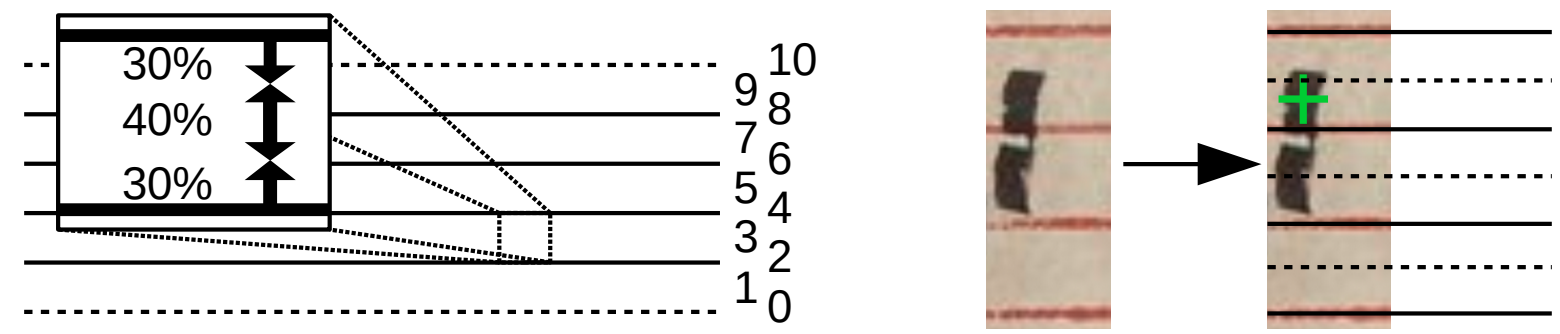

Figure 9. The left image shows the relative staff line positions shown on the right. The zoomed window marks the area sizes that are assigned as line or space, while the dashed lines indicate ledger lines. The right image is an example for a NC (green mark) that is visually closer to the space (dashed) however actually a NC on a line (solid).

To improve the quality of a line, we implemented and tested several pre-processing steps applied to the line image before being processed by the FCN (compare third to fifth image of Figure 7). First, we dewarp the line by transforming the image so that the staff lines are straight. Furthermore, we pad extra space to the left and right of a staff to ensure that all symbols especially clefs are fully contained. We optionally use data augmentation that varies the contrast and brightness up to a factor of $2^{[-0.1,0.1]}$ and a value of $4 \%$, respectively. Also, the data is scaled with a factor of up to $2^{[-0.1,0.1]}$ independently in $x$ - and $y$-direction.

\section{Experiments}

In this section, we first introduce the data setup which defines how we chose parts for training and testing the FCNs. Then, we evaluate and discuss the proposed staff line and symbol detection algorithm. Finally, we measure the computation times required for training the deep models and prediction.

\subsection{Data Setup}

In general, we report the average value of a five cross-fold as generated by the scheme shown in Figure 10. 
All

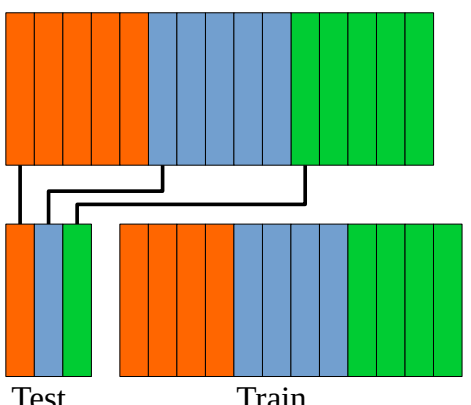

Test
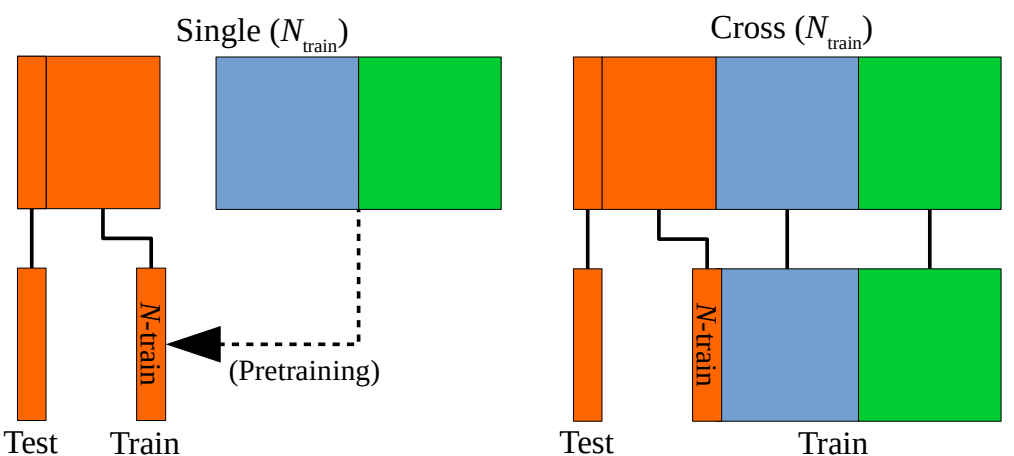

Figure 10. Scheme of the used cross fold training. Each color represents a part that is split into a five fold (smaller boxes). Depending on the experiment different sections are chosen for training and testing. Optionally, a pretrained model on remaining data is used. For the cross-part scheme $N_{\text {train }}$ can be set to zero to only train on other parts.

The different experimental setups are:

1. In the All approach, each part is split into a five fold whereof one fold of each part is chosen for testing, the remainder for training.

2. Only use a five fold of one single part. One fold is used for testing while $N_{\text {train }}$ data of the remainder serves for training. Optionally, a model pretrained on the two remaining parts can be utilised as starting point for the training on the actual part.

3. Cross-Fold: The same as experiment 2, however all pages of the remaining two parts are added to the training data. Setting $N_{\text {train }}$ to zero yields experiments that only incorporates data of the other parts and thus generate the models that are used for pretraining in the previous setup.

For both the staff line and symbol detection, setup 1 serves for a general evaluation of the proposed algorithm. Then, we use setup 3 with $N_{\text {train }}=0$ to test how a model generalised on unseen data with a somewhat different layout. Finally, setup 2 with and without pretraining and setup 3 with $N_{\text {train }} \geq 1$ conduce to test how the inclusion of actual data of the new part influences the accuracies.

\subsection{Staff Line Detection}

The staff line detection algorithm takes a pre-processed page image and outputs a list of staves each containing four staff lines stored as polyline. To evaluate this algorithm, we first introduce the used metrics and then present the results when training the FCN on different data sets. First, all parts are joined, then the impact of training on two and testing on the remaining one is investigated (compare Figure 10). Finally, we evaluate the effect of increasing the number of training examples.

\subsubsection{Metrics}

To evaluate the staff line detection algorithm we use two different pixel-based metrics. A true positive (TP) pixel pair of GT and prediction is allowed to have a distance of maximal $3 \mathrm{px}$, any other pixel of a line is treated as false positive (FP) or false negative (FN) (compare Figure 11).

Similar to an object detection task, we first measure the percentage of staff lines that are detected (recall) and if too many lines were found (precision), which then yields the line detection $F_{1}^{\mathrm{D}}$-score. A staff line is marked as TP if the overlap of GT and prediction is greater than 0.5 , that is more than $50 \%$ of the line must be hit, but the detected line may not be twice as long. This threshold is fairly harsh, since the detection of only a short section of a line causes both an FP and FN error.

The second metrics computes how many pixels of detected staff line are hit in length which indicates whether the prediction was too long or too short (compare Figure 11). Thereto, we evaluate the precision, recall and $F_{1}^{\mathrm{LF}}$-score of all TP-lines in the upper measure. Finally, multiplication of both individual metrics yields the total $F_{1}$-score. 
Furthermore, we evaluate the detection rate of a complete staff consisting of four staff lines $\left(F_{1}^{S}\right)$. A staff is marked as found if at least two staff lines $(=50 \%)$ are correctly detected. This arbitrary threshold is common for general object recognition tasks. For all detected staves we also count the relative amount of how many of the four lines are correctly hit $\left(F_{1}^{\mathrm{HL}}\right)$.

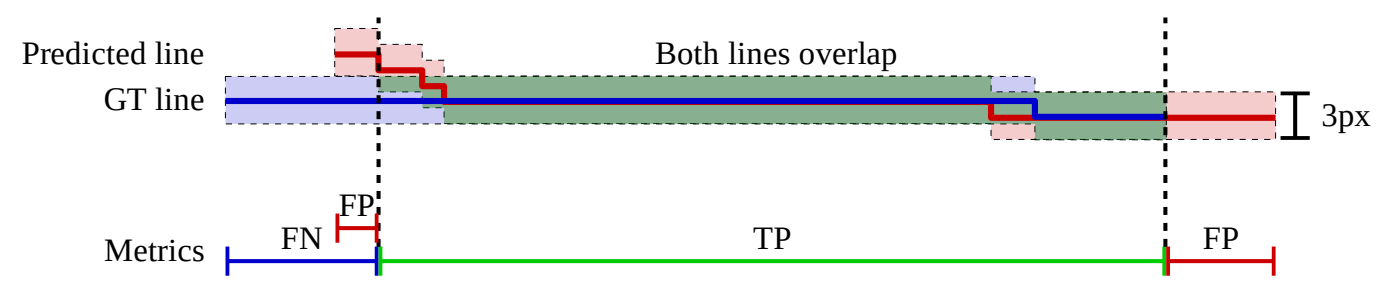

Figure 11. Visualisation of the metric used to evaluate the staff line detection. The blue and red solid lines in the upper image denote the GT and prediction of a single staff line, respectively. The colored area is the allowed space of 3 px where lines must overlap to be counted as a match. If at least one vertical pixel is green it is counted as correct. The bottom line shows the intervals which are counted as false negative (FN), true positive (TP) and false positive (FP). The deviation in the first part counts both as FN and FP.

\subsubsection{Results on All Data}

The results of the staff line detection using a five cross-fold on all available data are shown in Table 2.

Table 2. Staff line detection and length metrics with and without data augmentation. The last column combines the two individual $F_{1}$ measures by multiplication.

\begin{tabular}{cccccccc}
\hline & \multicolumn{3}{c}{ Line Detection } & \multicolumn{4}{c}{ Length Fit } \\
Model & Prec. & Rec. & $\boldsymbol{F}_{\mathbf{1}}^{\mathbf{D}}$ & Prec. & Rec. & $\boldsymbol{F}_{\mathbf{1}}^{\mathrm{LF}}$ & Total $\boldsymbol{F}_{\mathbf{1}}$ \\
\hline Default & 0.996 & 0.998 & 0.997 & 0.977 & 0.995 & 0.985 & 0.982 \\
Data aug. & 0.987 & 0.991 & 0.989 & 0.972 & 0.995 & 0.983 & 0.972 \\
\hline
\end{tabular}

Clearly over $99 \%$ of all staff lines are found (recall) and over $99 \%$ of all predicted staff lines are correct (precision). The resulting $F_{1}^{\mathrm{D}}$-score is about $99.7 \%$.

The length of the correctly detected staff lines has a $F_{1}^{\mathrm{LF}}$-score of $98.5 \%$; however, the recall that is higher than the precision shows that lines mostly are too long.

Typical errors are shown in Figure 12. The green, red and blue colors indicate TP, FP and FN pixels, respectively, though FN are rare. Most errors are either staff lines that are too long, for example caused by falsely extending a staff or staff lines that are predicted on background. Both error types are, among other things, caused by background noise such as bleeding staves or foreground text or drop capitals. Naturally, other errors occur on damaged pages or very thin lines that must almost be guessed. Furthermore, there are always small inaccuracies at the exact beginning and at the end of a staff line which however are also a problem of the GT itself. The severity of the errors on the symbol detection will be discussed in Section 5.3.5. 

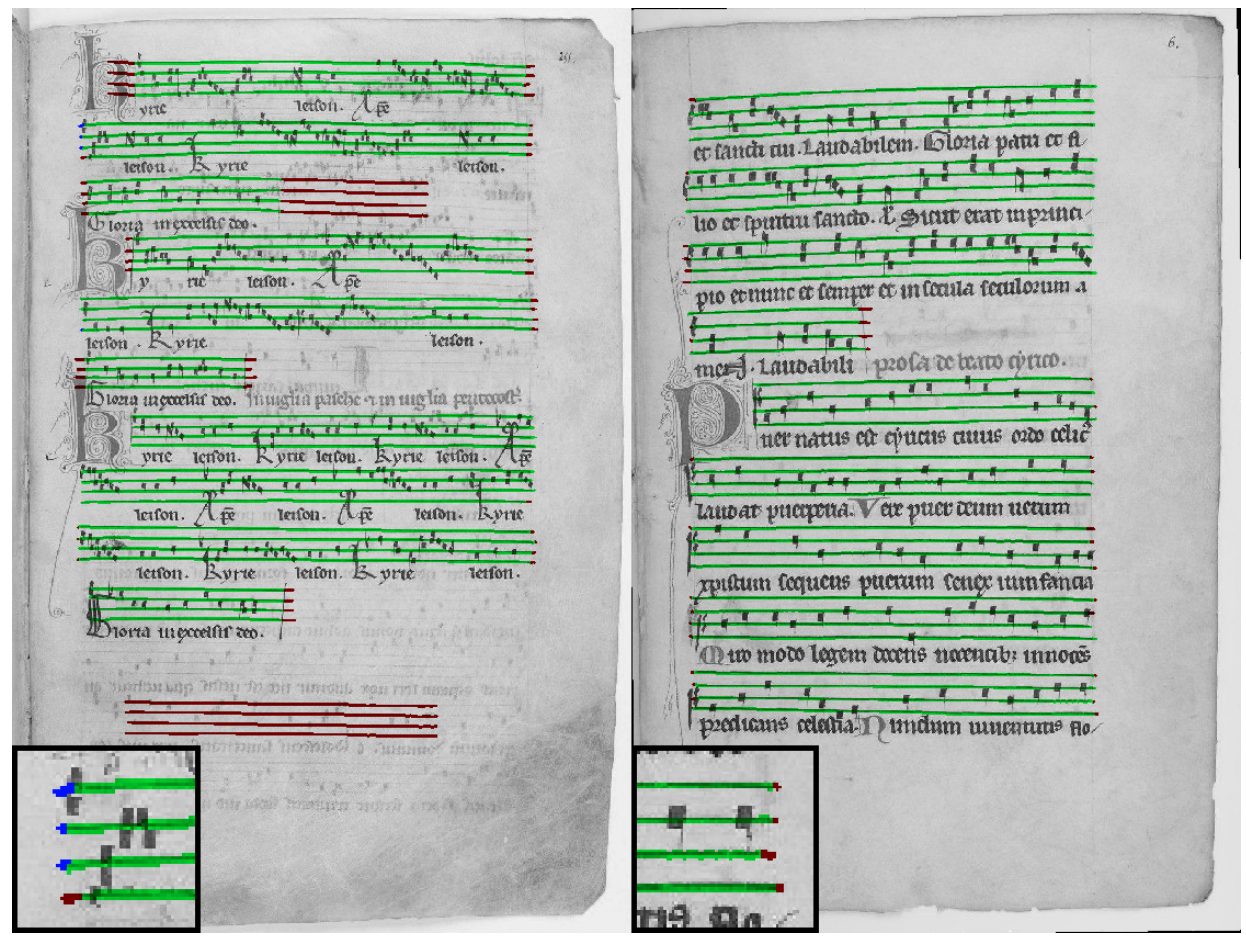

Figure 12. The left image shows typical errors during the staff line detection. Green pixels indicate TPs, red FPs, blue FNs. Either all lines in a staff are too long (third staff) or bled background is recognised (last staff). The right image shows a page with weak bleeding. In this image all staff lines are detected correctly. The zoomed sub images focus on the start and end of a staff.

Table 3 lists the recognition rate of complete staves. It is astonishing that the scores of the staff detection are (almost) identical to the staff line detection; however, this shows that the staff line detection only predicts wrong lines across a whole staff, for example all four lines are too long or a whole staff is predicted on a blank page, as seen in Figure 12. This is also shown by a precision and recall of $100 \%$ in the measure of hit lines. This states, that if a staff is detected all lines are correct. In the following, we will only report the staff line detection accuracy because it is almost equivalent to the staff accuracy but also includes the metric concerning the exact length of the lines.

Table 3. Staff detection and the number of recognised lines in a detected staff with and without data augmentation. The last column combines the two individual $F_{1}$ measures by multiplication.

\begin{tabular}{cccccccc}
\hline & \multicolumn{3}{c}{ Staff Detection } & \multicolumn{3}{c}{ Hit Lines } \\
Model & Prec. & Rec. & $\boldsymbol{F}_{\mathbf{1}}$ & Prec. & Rec. & $\boldsymbol{F}_{\mathbf{1}}^{\mathrm{HL}}$ & Total $\boldsymbol{F}_{\mathbf{1}}$ \\
\hline Default & 0.996 & 0.998 & 0.997 & 1.000 & 1.000 & 1.000 & 0.997 \\
Data aug. & 0.988 & 0.992 & 0.990 & 0.999 & 0.999 & 0.999 & 0.989 \\
\hline
\end{tabular}

Applying data augmentation yields no improvements in both metrics, instead the results clearly worsen. However, especially the precision drops compared to the recall. This is justified by errors that are mostly confusions if a staff is bleeding or real, which is possibly caused by the strong augmentations in contrast and brightness levels.

\subsubsection{Results of Cross-Part Training}

The results of cross-part training with and without data augmentation are shown in Table 4. 
Table 4. Comparing staff line detection using cross data set training: Two parts are chosen for training while the remainder is chosen as the test data set. The training data is split into five cross folds (four folds training, one for validation) of which the average scores are shown. The total score is computed as the product of the individual $F_{1}$-scores. The lower half of the table shows the results when using data augmentation.

\begin{tabular}{|c|c|c|c|c|c|c|c|c|c|}
\hline & \multirow[b]{2}{*}{ Train } & \multirow[b]{2}{*}{ Test } & \multicolumn{3}{|c|}{ Detection } & \multicolumn{3}{|c|}{ Length Fit } & \multirow[b]{2}{*}{ Total $F_{1}$} \\
\hline & & & Prec. & Rec. & $F_{1}^{\mathrm{D}}$ & Prec. & Rec. & $F_{1}^{\mathrm{LF}}$ & \\
\hline \multirow{4}{*}{$\underset{\infty}{\oplus}$} & 2,3 & 1 & 1.000 & 1.000 & 1.000 & 0.977 & 0.990 & 0.983 & 0.983 \\
\hline & 1,3 & 2 & 0.986 & 0.993 & 0.990 & 0.982 & 0.995 & 0.988 & 0.978 \\
\hline & 1,2 & 3 & 1.000 & 0.978 & 0.988 & 0.990 & 0.990 & 0.990 & 0.978 \\
\hline & \multicolumn{2}{|c|}{ Mean } & & & 0.993 & & & 0.987 & 0.980 \\
\hline \multirow{4}{*}{$\underset{\substack{D \\
0}}{P}$} & 2,3 & 1 & 1.000 & 1.000 & 1.000 & 0.976 & 0.993 & 0.984 & 0.984 \\
\hline & 1,3 & 2 & 0.959 & 0.978 & 0.968 & 0.968 & 0.997 & 0.982 & 0.951 \\
\hline & 1,2 & 3 & 0.989 & 0.967 & 0.977 & 0.988 & 0.995 & 0.991 & 0.969 \\
\hline & \multicolumn{2}{|c|}{ Mean } & & & 0.982 & & & 0.986 & 0.968 \\
\hline
\end{tabular}

In general, it can be observed that on any split our algorithm obtains an $F_{1}^{\mathrm{D}}$-score of $98.8 \%$ or higher in the staff line detection. On part 1 even $100 \%$ are reached, thus all staff lines are found with an overlap of at least $50 \%$. This shows that the staff line detection algorithm is very robust to new layouts. It is significant that part 2 suffers from data augmentation more clearly than part 3 , while on part 1 still a score of $100 \%$ is reached. The prediction of the line length is untouched. This can be explained by the different kind of quality of the parts. While part 3 suffers very weakly from bleeding staves and its lines are drawn very clearly, part 2 consists of pages where bleeding lines are present and some staff lines are barely visible and thus are more similar to background noise. Data augmentation creates, among other things, pages where the contrast of data is adjusted, which can explain why it is harder to distinguish actual staff lines, background and bleeding in part 2. In general, part 2 also yields the lowest $F_{1}^{\mathrm{D}}$-score of $96.8 \%$, which also shows that this part is the most complicated one.

The staff line lengths are detected very similar across the parts with a mean $F_{1}^{\mathrm{LF}}$-value of $98.6 \%$ and $98.7 \%$ (with and without data augmentation), which is almost identical to $F_{1}^{\mathrm{LF}}$ when training and evaluation on all data of $98.5 \%$ (compare Table 2). Hence, we conclude that the crucial component of the algorithm is to detect where staff lines are located and not to find the correct length of staff lines.

Note that the accuracies of these experiments are averaged over the parts while the results in the previous Table 3 are the mean of all pages, which is why the values should not be directly compared since part 2 consists of more instances than the other parts.

\subsubsection{Data Amount}

In this section, the effect of increasing the amount of training examples is investigated. The results are summarised in Table 5. Each value is computed by first averaging all cross-folds of a single part and then taking the mean of all three respective experiments for all parts.

As expected, if no additional data is available, an increasing number of training examples yields a higher $F_{1}$ score. The model trained on only one page already detects staves with $F_{1}^{\mathrm{D}}=98.3 \%$ and the length of a detected line matches with $F_{1}^{\mathrm{LF}}=96.9 \%$. Applying data augmentation on this data improves the results significantly, resulting in a $F_{1}^{\mathrm{D}}$ of over $99 \%$ and a $F_{1}^{\mathrm{LF}}$ of $98.0 \%$ if one page is used. Using more pages slightly increases the total $F_{1}$-score up to $97.9 \%$.

Of course using a pretrained model on the remaining data (PT) or including the remaining data into the data set (Inc.) clearly outperforms the standard training without data augmentation. However, it shows that including the data is in general better than just using a pretrained model if only one or two pages are available, which makes this approach more robust for few pages. If more data is used the error differences are caused by the training process itself for instance by choosing the random seed for initialisation of the model. But it is to be expected that if many GT pages are available PT 
is superior since the model can fit directly on the respective data. Data augmentation improves the results when PT is used, however not if the data is added to the dataset.

In general, it is remarkable that using $N_{\text {train }}=0$, that is, only using data of the other parts for training, yields very competitive results compared to any other experiment. Similar results are achieved if only training on one page of a single part but using data augmentation. Therefore, in practice it can be expected that the staff detection algorithm generalised well on unseen layouts.

Table 5. Evaluation how the number of training instances influence the accuracy of pages with an unknown layout. All results are the averages of a five fold of each data set part. Only $N_{\text {train }}$ pages are chosen for actual training. The major row grouping shows if pages of the remaining parts are included during training: Either no data is used (Default), a pretrained model on the data is used (PT), a large data set comprising all other data which is extended by $N_{\text {train }}$ is used (Inc.) The first row shows the cross-training (CT.) results of Table 4 without any data of the target layout. The two major column sections show the results with and without data augmentation on the training data. The best values for each $N_{\text {train }}$ are marked bold.

\begin{tabular}{cccccccc}
\hline & & \multicolumn{3}{c}{ No Data Augmentation } & \multicolumn{3}{c}{ Data Augmentation } \\
& $\boldsymbol{N}_{\text {train }}$ & $\boldsymbol{F}_{\mathbf{1}}^{\mathbf{D}}$ & $\boldsymbol{F}_{\mathbf{1}}^{\mathbf{L F}}$ & Tot. $\boldsymbol{F}_{\mathbf{1}}$ & $\boldsymbol{F}_{\mathbf{1}}^{\mathbf{D}}$ & $\boldsymbol{F}_{\mathbf{1}}^{\mathbf{L F}}$ & Tot. $\boldsymbol{F}_{\mathbf{1}}$ \\
\hline CT. & 0 & 0.993 & 0.987 & $\mathbf{0 . 9 8 0}$ & 0.982 & 0.986 & 0.968 \\
\hline \multirow{3}{*}{ Default } & 1 & 0.983 & 0.969 & 0.953 & 0.991 & 0.980 & 0.971 \\
& $\mathbf{2}$ & 0.986 & 0.976 & 0.962 & 0.990 & 0.985 & 0.975 \\
& 4 & 0.994 & 0.979 & 0.973 & 0.994 & 0.984 & 0.978 \\
& All & 0.989 & 0.984 & 0.974 & 0.994 & 0.986 & 0.979 \\
\hline \multirow{3}{*}{ PT } & 1 & 0.992 & 0.980 & 0.972 & 0.988 & 0.985 & 0.973 \\
& 2 & 0.992 & 0.981 & 0.973 & 0.993 & 0.984 & 0.977 \\
& 4 & 0.999 & 0.983 & $\mathbf{0 . 9 8 2}$ & 0.993 & 0.987 & 0.980 \\
Inc. & All & 0.995 & 0.984 & 0.980 & 0.998 & 0.986 & $\mathbf{0 . 9 8 4}$ \\
\hline & 1 & 0.992 & 0.988 & $\mathbf{0 . 9 8 0}$ & 0.985 & 0.988 & 0.973 \\
& 2 & 0.992 & 0.987 & $\mathbf{0 . 9 7 9}$ & 0.985 & 0.984 & 0.969 \\
& 4 & 0.994 & 0.986 & 0.980 & 0.986 & 0.986 & 0.972 \\
& All & 0.994 & 0.988 & 0.982 & 0.988 & 0.986 & 0.974 \\
\hline
\end{tabular}

\subsection{Symbol and Note Component Detection}

The symbol detection algorithm acts on extracted images that contain a single staff. To extract these images as described in Section 4.4, we generally used the GT staff lines to gain scores that are independent of the staff line accuracy, however in Section 5.3.5, we will also evaluate on predicted staff lines to investigate the impact of staff lines that are too short or too long. Similar to the staff line detection, we first present the used metrics. Afterwards, we determine different hyperparameters and settings for the algorithm. Finally, the outcomes of training on different parts and varying the number of training examples are shown.

\subsubsection{Metrics}

We use several metrics for the symbol detection evaluation. First, we measure both the detection rates of symbols in general $\left(F_{1}^{\text {all }}\right)$ and also the subgroups of notes $\left(F_{1}^{\text {note }}\right)$ and clefs $\left(F_{1}^{\text {clef }}\right)$. Thereto, we compare all GT and prediction symbols and match a pair as TP if two symbols are closer than 5px, all other symbols in the prediction and GT are treated as FP and FN, respectively. In the subgroups, we only account for symbols that match a note or clef in the GT or prediction. In the following, accidentals are not evaluated separately because no accidental is predicated at all.

Moreover, we measure how well types and staff locations of the note or clef subgroups are predicted. For each subgroup, our measure only takes TPs into account and counts the number of correctly predicted types $\left(A c c_{\text {type }}^{\text {note }}\right.$ and $\left.A c c_{\text {type }}^{\text {clef }}\right)$, that is the neume start and graphical connection for notes and the clef type ( $C$ or F) for clefs, or the staff positions ( $A c c_{\text {pos }}^{\text {note }}$ and $\left.A c c_{\text {pos }}^{\text {clef }}\right)$. 
Our last measure evaluates either the diplomatic or the harmonic transcription. We compare the produced sequence with the GT and count the number of insertions, deletions or substitutes in the sequence, which is similar to OCR tasks. As diplomatic transcription, we compare staff position and types of notes and clefs and their order; however, the actual horizontal position is ignored. Thus, only the resulting transcription is evaluated. The harmonic transcription is similar to the diplomatic one but ignores the type of notes, which is why only the harmonic information that is the melody is captured. We represent these two sequence accuracy rates by dSAR and hSAR.

\subsubsection{Preliminary Experiments}

The first experiments listed in Table 6 test variations of the hyperparameters. Thereto, we use the setup of Figure 10 where a cross-fold on all available data is taken. We list all scores, however the main focus lies on the general symbol detection, hSAR and dSAR, since these metrics do not act on subgroups but on the whole prediction.

Table 6. Metrics of various hyperparameter settings for the symbol detection. All experiments were conducted using a cross-fold of size five using all available data. The best values for each metric are highlighted.

\begin{tabular}{lccccccccc}
\hline & \multicolumn{3}{c}{ Detection } & \multicolumn{2}{c}{ Type } & \multicolumn{2}{c}{ Position in Staff } & \multicolumn{2}{c}{ Sequence } \\
& $\boldsymbol{F}_{\mathbf{1}}^{\text {all }}$ & $\boldsymbol{F}_{\mathbf{1}}^{\text {note }}$ & $\boldsymbol{F}_{\mathbf{1}}^{\text {clef }}$ & $A c c_{\text {type }}^{\text {note }}$ & $A c c_{\text {type }}^{\text {clef }}$ & $A c c_{\text {pos }}^{\text {note }}$ & $A c c_{\text {pos }}^{\text {clef }}$ & hSAR & dSAR \\
\hline$h_{\text {staff }}=40$ & 0.926 & 0.936 & 0.523 & 0.929 & $\mathbf{0 . 9 2 5}$ & 0.963 & 0.992 & 0.834 & 0.792 \\
$h_{\text {staff }}=60$ & 0.953 & 0.961 & 0.655 & 0.944 & 0.911 & 0.968 & 0.994 & 0.877 & 0.844 \\
$h_{\text {staff }}=80$ & $\mathbf{0 . 9 5 6}$ & 0.964 & $\mathbf{0 . 6 9 3}$ & 0.949 & 0.897 & $\mathbf{0 . 9 7 0}$ & 0.990 & $\mathbf{0 . 8 8 5}$ & $\mathbf{0 . 8 5 6}$ \\
$h_{\text {staff }}=100$ & $\mathbf{0 . 9 5 6}$ & $\mathbf{0 . 9 6 5}$ & 0.673 & $\mathbf{0 . 9 5 0}$ & 0.911 & $\mathbf{0 . 9 7 0}$ & $\mathbf{0 . 9 9 5}$ & 0.884 & $\mathbf{0 . 8 5 6}$ \\
\hline Pad & 0.962 & 0.966 & $\mathbf{0 . 8 2 9}$ & 0.949 & $\mathbf{0 . 9 8 2}$ & 0.967 & 0.990 & 0.893 & 0.866 \\
Dewarp & 0.952 & 0.959 & 0.708 & 0.946 & 0.873 & 0.969 & 0.987 & 0.877 & 0.845 \\
Aug. & 0.959 & 0.968 & 0.671 & $\mathbf{0 . 9 5 3}$ & 0.912 & 0.970 & $\mathbf{0 . 9 9 2}$ & 0.889 & 0.862 \\
Pad, Aug. & $\mathbf{0 . 9 6 4}$ & $\mathbf{0 . 9 6 9}$ & 0.818 & $\mathbf{0 . 9 5 2}$ & $\mathbf{0 . 9 8 2}$ & $\mathbf{0 . 9 7 1}$ & $\mathbf{0 . 9 9 2}$ & $\mathbf{0 . 8 9 8}$ & $\mathbf{0 . 8 7 1}$ \\
\hline
\end{tabular}

The upper block shows the results when the resolution of the input line is varied. The bold numbers highlighting the best value for each score indicate that the accuracies seem to stagnate, which is why in the following we use a line height of $80 \mathrm{px}$.

Using this hyperparameter, we evaluated the outcome of using padding, dewarping and data augmentation. Since padding is designed to add space to the left of a staff particularly where clefs are located, the highest score of clef type detection are achieved. As a result, the overall accuracies improve as well. Dewarping yields slightly worse results in any metric, which is why in the following, it was omitted in each experiment. We explain this unexpected behavior with the induced distortions which complicates the detection of notes and especially their connection. Data augmentation slightly improved the results compared to the default model. Since $80 \%$ of all data is used for training it is to be expected, that data augmentation improves the results more clearly if less data is used for training. We show this behavior in Section 5.3.4. The last row shows the best overall result reached when combining data augmentation, padding, and choosing a line height of $80 \mathrm{px}$.

\subsubsection{Results of Cross-Part Training}

Table 7 show the result of cross-part training, that is testing on one of the three parts and training on the two remaining ones. In general, as expected, data augmentation improves the results. The ranking of accuracies follow the quality of the data: in summary, the highest values in $F_{1}^{\text {all }}$, hSAR, and dSAR are achieved on part 1 , followed closely by part 3 , and finally part 2 . The similar accuracies reached on part 1 and 3 in almost all metrics show the greater similarities of these two parts and in general a clearer notation compared to part 2. 
Table 7. Symbol detection scores when testing on one and training on the remaining parts. The upper and lower rows show the results without and with data augmentation, respectively. Padding and a line height of 80px are used for all experiments.

\begin{tabular}{|c|c|c|c|c|c|c|c|c|c|c|c|}
\hline & \multirow[b]{2}{*}{ Test } & \multirow[b]{2}{*}{ Train } & \multicolumn{3}{|c|}{ Detection } & \multicolumn{2}{|c|}{ Type } & \multicolumn{2}{|c|}{ Position in Staff } & \multicolumn{2}{|c|}{ Sequence } \\
\hline & & & $F_{1}^{\text {all }}$ & $F_{1}^{\text {note }}$ & $F_{1}^{\text {clef }}$ & $A c c_{\text {type }}^{\text {note }}$ & $A c c_{\text {type }}^{\text {clef }}$ & $A c c_{\text {pos }}^{\text {note }}$ & $A c c_{\text {pos }}^{\text {clef }}$ & hSAR & dSAR \\
\hline \multirow{4}{*}{ Def. } & 1 & 2,3 & 0.956 & 0.965 & 0.782 & 0.914 & 1.000 & 0.981 & 0.993 & 0.906 & 0.860 \\
\hline & 2 & 1,3 & 0.912 & 0.916 & 0.638 & 0.928 & 0.975 & 0.955 & 0.990 & 0.797 & 0.757 \\
\hline & 3 & 1,2 & 0.950 & 0.952 & 0.907 & 0.958 & 0.968 & 0.981 & 1.000 & 0.895 & 0.867 \\
\hline & \multicolumn{2}{|c|}{ Mean } & 0.939 & 0.944 & 0.776 & 0.933 & 0.981 & 0.972 & 0.994 & 0.866 & 0.828 \\
\hline \multirow{4}{*}{ Aug. } & 1 & 2,3 & 0.958 & 0.964 & 0.822 & 0.914 & 1.000 & 0.980 & 0.990 & 0.907 & 0.862 \\
\hline & 2 & 1,3 & 0.939 & 0.945 & 0.699 & 0.947 & 0.975 & 0.965 & 0.990 & 0.848 & 0.817 \\
\hline & 3 & 1,2 & 0.943 & 0.945 & 0.901 & 0.959 & 0.956 & 0.981 & 1.000 & 0.886 & 0.859 \\
\hline & \multicolumn{2}{|c|}{ Mean } & 0.946 & 0.951 & 0.807 & 0.940 & 0.977 & 0.976 & 0.993 & 0.881 & 0.846 \\
\hline
\end{tabular}

The dSAR of any part is considerably lower than the value of $86.6 \%$ and $87.1 \%$ yielded when training on all data (compare Table 6) with and without data augmentation, respectively. Also the ratio of detected symbols $F_{1}^{\text {all }}$ is significantly lower, due to the variations in style across the parts. Acc pose and $A c c_{\text {pos }}^{\text {clef }}$ are similar to training on all data which shows that if a symbol is correctly detected, its position on the staff lines is usually correct. This behavior is expected because these errors are almost always intrinsic ambiguities which are independent of the layout. Furthermore, the average clef type accuracy $A c c_{\text {type }}^{\text {clef }}$ of $97.7 \%$ in average is also very high. Therefore the network is mostly certain if a $\mathrm{C}$ - or F-clef is written even though they share similarities. The decision whether a NC is graphically or logically connected is correct in only $94.0 \%$ of all detected notes and slightly lower than the $95.2 \%$ reported in Table 6. This shows that the FCN can transfer information about the arrangement of NCs that build typical neumes.

\subsubsection{Varying the Number of Training Examples}

In this section, we also train on two parts and test on the remaining part, however actual data of the target part is included. Table 8 lists the results when using $0,1,2,4$, or finally all pages.

Table 8. Symbol detection scores when varying the number of training examples. The first line is the average of the cross-part training (CT.) experiment of Table 7 where no pages of the target part are used. The next rows (Default) only use $N_{\text {train }}$ of the target data set for training. Afterwards, a pretrained model is used (PT.) or the additional part data is included (Inc.).

\begin{tabular}{ccccccccccc}
\hline & & \multicolumn{3}{c}{ Detection } & \multicolumn{2}{c}{ Type } & \multicolumn{2}{c}{ Position in Staff } & \multicolumn{2}{c}{ Sequence } \\
& $N_{\text {train }}$ & $\boldsymbol{F}_{\mathbf{1}}^{\text {all }}$ & $\boldsymbol{F}_{\mathbf{1}}^{\text {note }}$ & $\boldsymbol{F}_{\mathbf{1}}^{\text {clef }}$ & $A c c_{\text {type }}^{\text {note }}$ & $A c c_{\text {type }}^{\text {clef }}$ & $A c c_{\text {pos }}^{\text {note }}$ & $A c c_{\text {pos }}^{\text {clef }}$ & hSAR & dSAR \\
\hline CT. & 0 & 0.946 & 0.951 & 0.807 & 0.940 & 0.977 & 0.976 & 0.993 & 0.881 & 0.846 \\
\hline \multirow{3}{*}{ Default } & 1 & 0.846 & 0.854 & 0.533 & 0.862 & 0.963 & 0.954 & 0.986 & 0.730 & 0.661 \\
& 2 & 0.899 & 0.905 & 0.706 & 0.901 & 0.989 & 0.962 & 0.998 & 0.806 & 0.749 \\
& 4 & 0.926 & 0.930 & 0.775 & 0.927 & 0.982 & 0.970 & 0.990 & 0.846 & 0.804 \\
& All & 0.954 & 0.958 & 0.861 & 0.945 & 0.987 & 0.977 & 0.998 & 0.897 & 0.867 \\
\hline \multirow{3}{*}{ PT. } & 1 & 0.925 & 0.929 & 0.760 & 0.907 & 0.978 & 0.956 & 0.992 & 0.834 & 0.777 \\
& 2 & 0.939 & 0.942 & 0.796 & 0.928 & 0.981 & 0.966 & 0.999 & 0.862 & 0.821 \\
& 4 & 0.953 & 0.956 & 0.871 & 0.944 & 0.987 & 0.972 & 0.996 & 0.890 & 0.858 \\
Inc. & All & 0.969 & 0.971 & 0.910 & 0.950 & 0.989 & 0.975 & 0.997 & 0.916 & 0.888 \\
\hline & 1 & 0.948 & 0.953 & 0.808 & 0.936 & 0.982 & 0.977 & 0.997 & 0.882 & 0.846 \\
& 2 & 0.952 & 0.958 & 0.793 & 0.939 & 0.978 & 0.976 & 0.997 & 0.887 & 0.854 \\
& 4 & 0.959 & 0.964 & 0.817 & 0.948 & 0.980 & 0.976 & 0.996 & 0.900 & 0.870 \\
& All & 0.961 & 0.966 & 0.846 & 0.950 & 0.979 & 0.977 & 0.996 & 0.905 & 0.878 \\
\hline
\end{tabular}


As expected, an increasing number of training examples leads in general to improve recognition rates and thus higher scores in any experiment. However, it is remarkable that the model which only comprises one page without any other techniques already yields an dSAR of $66.1 \%$ and a hSAR of $73.0 \%$. Nevertheless, the scores significantly improve if other data are incorporated even if no page of the target part is included at all $\left(N_{\text {train }}=0\right)$, yielding a dSAR of $84.6 \%$ and a hSAR of $88.1 \%$. Only if all available pages are used for training $(\mathrm{dSAR}=86.7 \%)$, the default model is slightly better than training on the other parts only $(\mathrm{dSAR}=84.6 \%)$.

However, there are huge differences when to use pretraining or data inclusion. If only few data of the target set are available $(1,2,4)$ including the data yields better results but if many examples are used during training, PT is superior. The reason is that the PT model yields a good starting point which is then optimised for one specific part if enough target data is used. Whereas, if all data is included, the model is forced to generalise on any part and not on a specific one.

Furthermore, the experiments show that the accuracy of the position in staff increase with a higher number of training instances in the default model or when using PT but are almost constant if zero or all instances are added to the training set. Obviously, having more data available it is easier to find and remember good rules that state how to resolve incertitudes.

On the whole, the model training if no data of the target part already yields a very robust $F_{1}^{\text {all }}$ and dSAR-score. Even if PT and all data is used the dSAR increases only from $88.1 \%$ to $91.5 \%$, which is a relative improvement of about $4 \%$. However, we highly expect that if the differences between training and testing data are even more clear, the effect will increase.

\subsubsection{Symbol Detection on Predicted Staves}

Finally, we test the combination of both algorithms. Thus, in this experiment, the impact of shortened or too long staff lines of the predicted staves can be investigated. Thereto, we only evaluated on staves that are marked as TPs which is why for an combined result the amount of detected staves must be included but which is almost 1 . We evaluate the model which is trained on a cross-fold of all pages combined.

The experiment yields a hSAR of $88.9 \%$ and a dSAR of $86.2 \%$, which is slightly worse than the results obtained if predicted on the corrected lines ( $89.8 \%$ and $87.1 \%$ respectively). The sole new errors that can occur are FPs if the staves are expanded into other content such as drop capitals or text, or FNs if the staves are too short.

In conclusion, the dSAR decreases by only $1 \%$ on detected staves due to wrong staff lengths. The score is further reduced by approximately $1 \%$ when accounting for FPs and FNs during the staff prediction.

Figure 13 shows the full output of the symbol detection acting on recognised staves. Hereby, all symbol positions are mapped relative to the page by transforming the prediction of the symbol detection to global space. The various error sources are discussed in Section 5.3.6. 


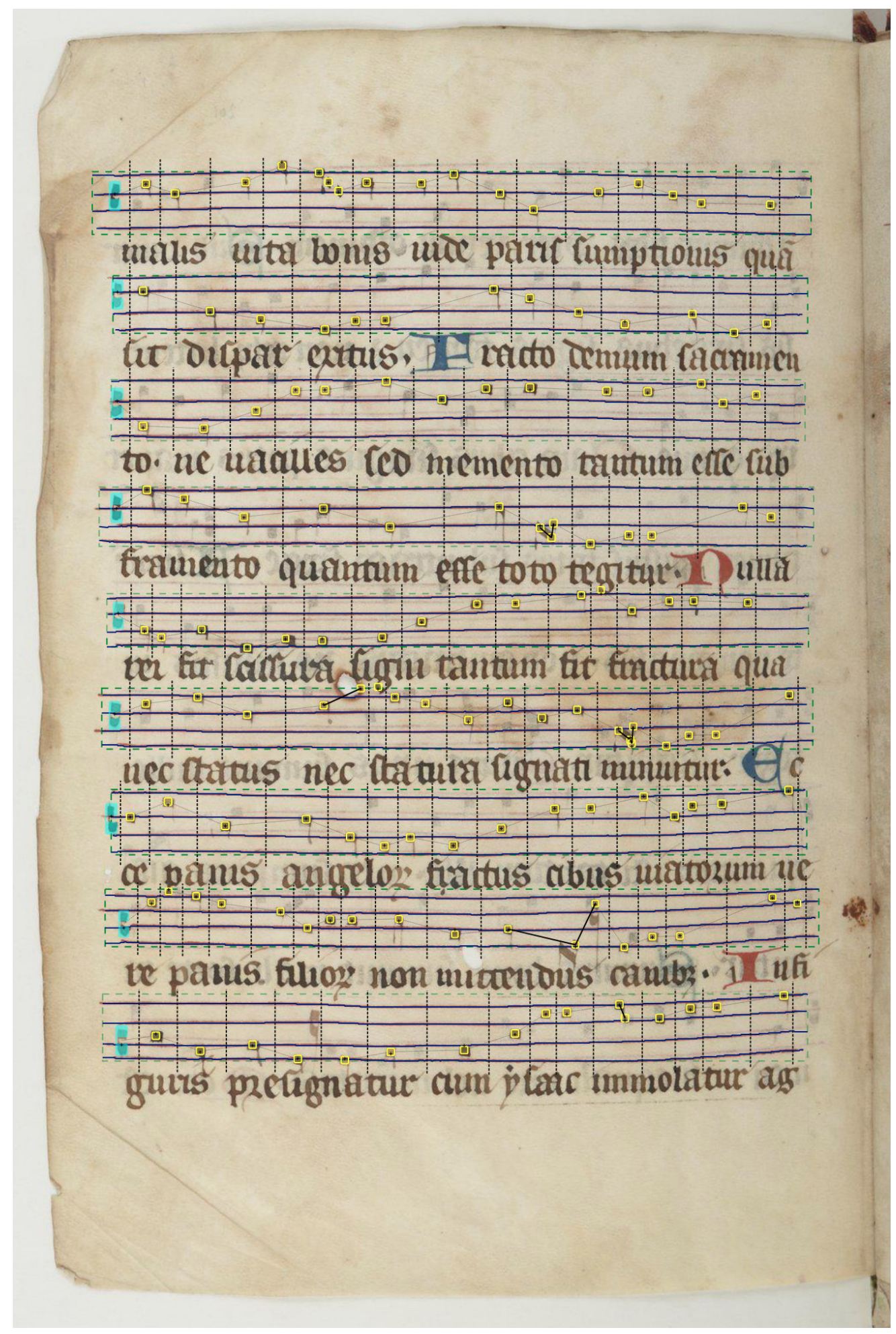

Figure 13. The page visualises the full prediction of the staff line and symbol detection. Encoded staff lines are drawn as blue lines on top of the red staff lines of the original manuscript, the green dashed lines mark the bounding boxes of single staves. The vertical dashed lines separate neumes whose NCs are drawn as yellow boxes. Graphical connection withing a neume are indicated by a black stroke between two NCs. The reading order is represented by a thin dotted line that connects all NCs withing a staff. Small crosses inside of the yellow box of a NCs mark the discrete location of the symbol relative to the staff lines. The cyan symbols mark clefs. 


\subsubsection{Error Analysis}

Regarding the error analysis, we compare the predicted and GT sequences and count how many deletions or insertions of a specific type are required to gain the GT. We use the model, when training and evaluating a cross fold comprising all data, which yields a dSAR of $87.1 \%$ (compare Table 6). Note that in this section replacements are treated as two errors: one insertion and one deletion. Table 9 lists eight groups of errors and their relative impact on the total dSAR whereas Figure 14 shows examples for the errors.

Table 9. Error analysis: The relative amount of errors of the output sequence is categorised.

\begin{tabular}{rlr}
\hline \multicolumn{2}{c}{ Group } & Error \\
\hline False Negatives & $51.7 \%$ \\
1. & Missing Note & $21.2 \%$ \\
2. & Wrong Connection & $10.8 \%$ \\
3. & Wrong Location & $14.3 \%$ \\
4. & Missing Clef & $3.5 \%$ \\
5. & Missing Accidental & $1.9 \%$ \\
\hline False Positives & $48.3 \%$ \\
6. & Additional Note & $20.6 \%$ \\
2. & Wrong Connection & $10.8 \%$ \\
3. & Wrong Location & $14.3 \%$ \\
7. & Additional Clef & $2.6 \%$ \\
8. & Additional Accidental & $0.0 \%$ \\
\hline & Total & $100 \%$ \\
\hline
\end{tabular}

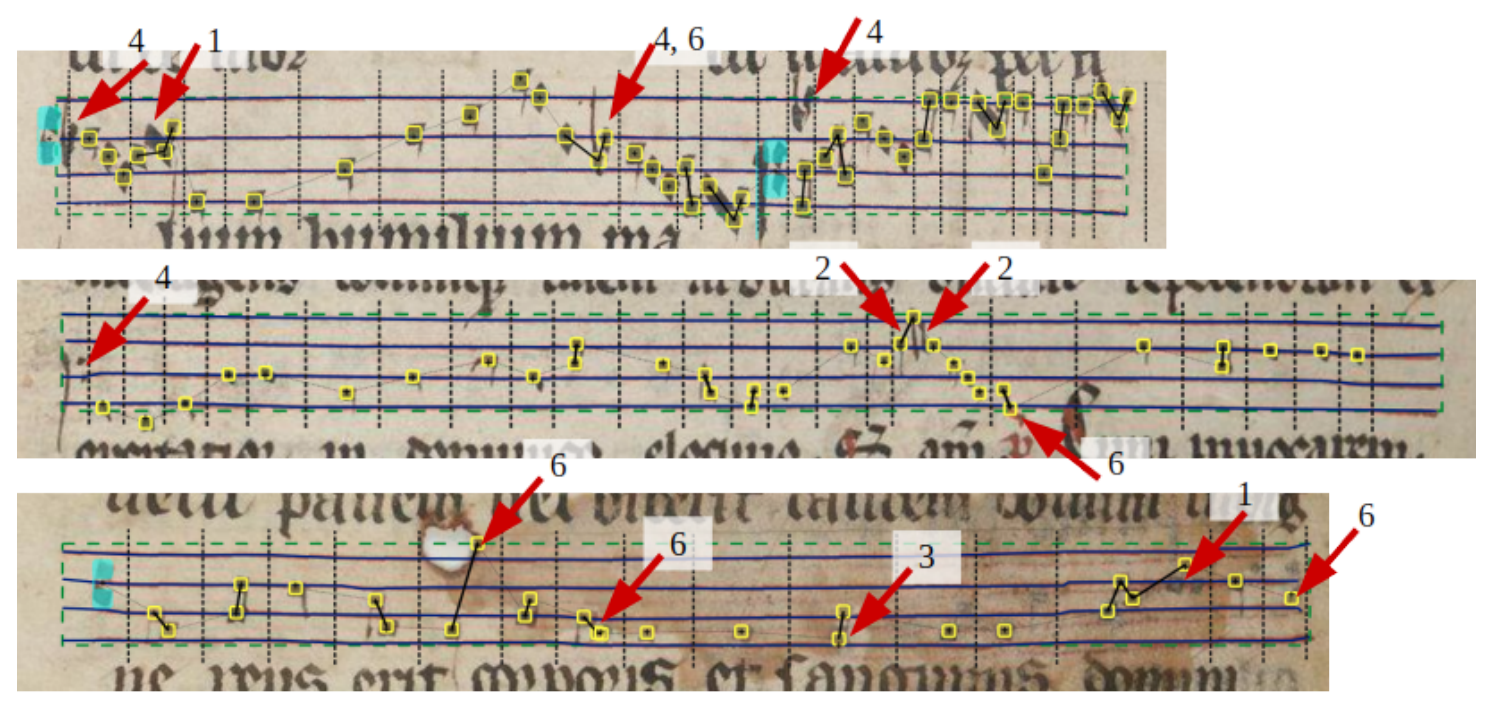

Figure 14. The three lines exemplarily show typical errors of the symbol detection algorithm. Refer to Table 9 for the definition of the numbers indicating different groups. The yellow boxes mark single NCs, solid lines between two NCs indicate that the second note is graphically connected to the previous. The dashed vertical lines separate single neumes and therefore represent logical connections. The cyan symbols denote clefs. The connecting thin dotted line represents the reading order of the symbols.

The first result is that the combined amount of FPs and FNs are balanced (48\% vs. $52 \%)$. Notes are missed (1) most often if the contrast between notes and background is too low or if neumes are written very dense, which also significantly affects the prediction accuracy of connection types (2). Wrong note positions (3) occur only at locations where even for a human it is difficult to decide whether the $\mathrm{NC}$ is located on a line or in a space. Mainly neumes with stacked NCs, such as a so-called pes (first neume in Figure 1) or scandicus, suffer from these errors. These neumes also induce errors in reading 
order which is only captured by SAR metrics. Since the NCs are ordered left to right based on their prediction position, it happens that the upper note, which should be sung later, is predicted a little to far to the left compared to the lower note. Observations show that the first clef in a staff was almost always detected, whereas a clef in a line was very often missed (4) and sometimes misrecognised as two notes. Accidentals are not detected at all (5) possibly due to their underrepresentation in the available data set.

Additional notes (6) mostly occur if clefs or accidentals are labeled as two notes or if noise or bleeding is falsely detected as symbols. Wrong connections and locations count also as FP which is why their ratio is the same. The amount of FPs that are clefs (7) is very low and since no accidentals are predicted at all no FPs (8) can occur.

In conclusion, it can be observed that the majority of errors are, as expected, note related; however, missing $(21.2 \%)$, additional $(20.6 \%)$, wrong connection (21.6\%) and wrong location $(28.6 \%)$ errors are roughly balanced.

\subsection{Timing}

Finally, we measured the times required for predicting staff lines or the symbols of a staff line including data loading and pre- and postprocessing steps (see Table 10). The time for loading the model which is only required once is included. All times were measured on a Nvidia Titan X GPU and an Intel Core i7-5820K processor, while the FCN was implemented in Tensorflow. Moreover, we obtained the results only when using the CPU or a GPU if available.

Table 10. Prediction and training times in seconds. For the staff line detection the numbers denote the time to process a whole page, while for the symbol detection the reference is a single staff. Prediction includes pre- and postprocessing; however, training only refers to a single iteration.

\begin{tabular}{ccccc}
\hline & \multicolumn{2}{c}{ Staff Line } & \multicolumn{2}{c}{ Symbols } \\
& GPU & CPU & GPU & CPU \\
\hline Predict & 3.5 & 3.7 & 0.15 & 0.12 \\
Train & 0.12 & 3.6 & 0.04 & 0.42 \\
\hline
\end{tabular}

To process a full page the staff line detection requires 3.7 (CPU) and 3.5 (GPU) seconds, therefore about $3 \mathrm{~min}$ for the full dataset. The pre-processing step, which normalises the input image, takes about half of the time. The application of the FCN follows with about $40 \%$, however the difference between CPU and GPU is minor. This can be explained by the shorter model loading time when using the CPU and the faster computation time but a required data transfer when using the GPU. The remaining time is consumed by the postprocessing which generates the final output.

The symbol detection requires about 0.12 (CPU) and 0.15 (GPU) seconds for the prediction of a single staff, thus the complete dataset takes one minute to predict. Because no pre-processing besides cutting the image is required and the postprocessing step only consists of a connected component analysis, it is clearly faster than the pre- and postprocessing of the staff line detection, yet it is the most time consuming step. Compared to the staff detection, the CPU is even a bit faster due to the smaller input line image. In total about 4 minutes are required to predict both the staves and symbols on all 49 pages.

Especially for training, GPU usage is almost indispensable to enable a fast back-propagation which computes the weight differences required for training the network. Compared to the CPU, the staff line training on the GPU that acts on the full page yields a speed-up of 30 , which is justified with the convolution and pooling operations of the FCN that are highly optimised and perfectly suited for the GPU. Note that training a single instance is faster than prediction because pre- and postprocessing steps are not required. 


\section{Conclusion and Future Work}

This paper introduces a new system for automatic staff line and symbol detection on historical medieval notations based on deep neural networks. The main results on our data set are (compare Table 11):

Table 11. Overview of the main results of this paper.

\begin{tabular}{lc}
\hline Task & Score \\
\hline$F_{1}$-scores of staff line \& staff detection & $>99 \%$ \\
$F_{1}$-score of detected Symbols & $>96 \%$ \\
Diplomatic sequence accuracy (dSAR) & $\approx 87 \%$ \\
\hline
\end{tabular}

- Staff lines and staves are recognised with an $F_{1}$-score greater than $99 \%$. The major error sources are staves that are detected on a bled background or which are extended into other foregrounds (e.g., text).

- A trained staff line model generalises flawlessly to different layout types and thus needs no retraining.

- $\quad$ Our best model finds symbols with an $F_{1}$-score of above $96 \%$ and yields a dSAR of about $87 \%$.

- Pretraining is helpful but not mandatory for the symbol detection, since the dSAR only increases about $4 \%$.

- The full transcription (compare Figure 13) comprises staves consisting of four staff lines stored as polylines, the position and type of clefs, the position of accidentals, the position and graphical connection of NCs which comprise neumes.

To further improve the staff detection, which is close to perfect, more experiments must be conducted to evaluate the effect of data augmentation which is unclear in the proposed manner. Augmentations in brightness and contrast must especially be reconsidered to allow for more robust models. Furthermore, staves that are detected on background can be dropped by introducing a threshold of minimum symbols per staff because the symbol detection only occasionally detects symbols on background. Another problem are staves that extend into text or drop capitals. However a symbol detection which is explicitly trained to ignore text could help to determine the boundaries between staff and text because no symbols should be detected there.

However, the primary goal of our future work is to further reduce the errors of the symbol detection. First, the error analysis shows that false notes are the main source of symbol errors. The connection and location of notes induces many errors, which however are also difficult tasks for humans. We proposed to evaluate a two stage approach similar to Reference [7] that first predict neumes as combined components and then separates them into individual NCs; however, with incorporation of deep neural networks for object detection. Furthermore, to improve the quality of clef or accidental prediction more data must be gathered or over-sampled in the training data either by data augmentation or by presenting these lines more often.

A completely different approach, which is based on the state-of-the-art networks in OCR, is to directly use sequence-to-sequence networks as already proposed in Reference [28] on contemporary OMR. A CNN/LSTM hybrid network with a combination of a CTC-loss function directly predicts an NC sequence. The main advantage is that GT production is easier since only the sequence has to be transcribed but not the actual position of each single NC. However, it is to be expected that more GT is required since it has to learn autonomously both the shape of all music symbols and the staff lines to infer the location of the symbols. It is still an open issue whether this CTC-approach in fact yields improved results. Furthermore, because this approach directly predicts a symbol sequence and no actual note positions relative to the image, it cannot be used if this information is mandatory. 
Author Contributions: C.W. conceived and performed the experiments and created the GT data. C.W. and A.H. contributed the staff line detection algorithm. C.W. designed the symbol detection algorithm. C.W. and F.P. analysed the results. C.W. wrote the paper with substantial contributions of F.P.

Funding: This research received no external funding.

Acknowledgments: We would like to thank Tim Eipert for helping to resolve ambiguities in the data set.

Conflicts of Interest: The authors declare no conflict of interest.

$\begin{array}{ll}\text { Abbreviations } \\ \text { The following abbreviations are used in this ma } \\ \text { CNN } & \text { Convolutional Neural Network } \\ \text { CPU } & \text { Central Processing Unit } \\ \text { CTC } & \text { Connectionist Temporal Classification } \\ \text { dSAR } & \text { Diplomatic Sequence Accuracy Rate } \\ \text { FCN } & \text { Fully Convolutional Network } \\ \text { FN } & \text { False Negative } \\ \text { FP } & \text { False Positive } \\ \text { hSAR } & \text { harmonic Sequence Accuracy Rate } \\ \text { LSTM } & \text { Long Short-Term Memory } \\ \text { GPU } & \text { Graphical Processing Unit } \\ \text { GT } & \text { Ground Truth } \\ \text { NC } & \text { Note Component } \\ \text { OCR } & \text { Optical Character Recognition } \\ \text { OMR } & \text { Optical Music Recognition } \\ \text { PT } & \text { Pretraining } \\ \text { RLE } & \text { Run Length Encoding } \\ \text { TP } & \text { True Positive }\end{array}$

\section{References}

1. Good, M. MusicXML for notation and analysis. In The Virtual Score: Representation, Retrieval, Restoration; MIT Press: Cambridge, MA, USA, 2001; Volume 12, pp. 113-124.

2. Hankinson, A.; Burgoyne, J.A.; Vigliensoni, G.; Fujinaga, I. Creating a large-scale searchable digital collection from printed music materials. In Proceedings of the 21st International Conference on World Wide Web, Lyon, France, 16-20 April 2012; pp. 903-908.

3. Hild, E.S. Tropen zu den Antiphonen der Messe aus Quellen französischer Herkunft; Corpus Monodicum. Die einstimmige Musik des lateinischen Mittelalters, Schwabe Verlag: Basel, Switzerland, 2016.

4. Haug, A.; Kraft, I.; Zühlke, H. Tropen zu den Antiphonen der Messe aus Quellen deutscher Herkunft; Corpus Monodicum. Die einstimmige Musik des lateinischen Mittelalters, Schwabe Verlag: Basel, Switzerland, 2019.

5. Corbin, S.; Institut, B.M. Die Neumen; Palaeographie der Musik. Bd. 1, Fasz. 3; Arno Volk-Verlag: Köln, Germany, 1977.

6. Vigliensoni, G.; Burgoyne, J.A.; Hankinson, A.; Fujinaga, I. Automatic pitch recognition in printed square-note notation. In Proceedings of the ISMIR, Miami, FL, USA, 24-28 October 2011.

7. Ramirez, C.; Ohya, J. Automatic recognition of square notation symbols in western plainchant manuscripts. J. New Music Res. 2014, 43, 390-399. [CrossRef]

8. Baró, A.; Riba, P.; Calvo-Zaragoza, J.; Fornés, A. Optical Music Recognition by Long Short-Term Memory Networks. In Graphics Recognition. Current Trends and Evolutions; Springer International Publishing: Cham, Switzerland, 2018; pp. 81-95.

9. Keil, K.; Ward, J.A. Applications of RISM data in digital libraries and digital musicology. Int. J. Digit. Libr. 2019, 20, 3-12. [CrossRef]

10. Fornés, A.; Dutta, A.; Gordo, A.; Lladós, J. CVC-MUSCIMA: A ground truth of handwritten music score images for writer identification and staff removal. Int. J. Doc. Anal. Recognit. 2012, 15, 243-251. [CrossRef] 
11. Van der Wel, E.; Ullrich, K. Optical Music Recognition with Convolutional Sequence-to-Sequence Models. In Proceedings of the 18th International Society for Music Information Retrieval Conference, ISMIR 2017, Suzhou, China, 23-27 October 2017; Cunningham, S.J., Duan, Z., Hu, X., Turnbull, D., Eds.; 2017; pp. 731-737.

12. Sutskever, I.; Vinyals, O.; Le, Q.V. Sequence to sequence learning with neural networks. In Proceedings of the 27th International Conference on Neural Information Processing Systems-Volume 2, Montreal, QC, Canada, 8-13 December 2014; pp. 3104-3112.

13. Calvo-Zaragoza, J.; Rizo, D. End-to-end neural optical music recognition of monophonic scores. Appl. Sci. 2018, 8, 606. [CrossRef]

14. Graves, A.; Fernández, S.; Gomez, F.; Schmidhuber, J. Connectionist temporal classification: labelling unsegmented sequence data with recurrent neural networks. In Proceedings of the 23rd International Conference on Machine Learning, Pittsburgh, PA, USA, 25-29 June 2006; pp. 369-376.

15. Breuel, T.M. High Performance Text Recognition Using a Hybrid Convolutional-LSTM Implementation. In Proceedings of the 14th IAPR International Conference on Document Analysis and Recognition, ICDAR 2017, Kyoto, Japan, 9-15 November 2017; pp. 11-16. [CrossRef]

16. Wick, C.; Reul, C.; Puppe, F. Comparison of OCR Accuracy on Early Printed Books using the Open Source Engines Calamari and OCRopus. JLCL 2018, 33, 79-96.

17. Song, W.; Cai, J. End-to-end Deep Neural Network for Automatic Speech Recognition; Standford CS224D Reports; Stanford University: Stanford, CA, USA, 2015.

18. Zhang, Y.; Chan, W.; Jaitly, N. Very deep convolutional networks for end-to-end speech recognition. In Proceedings of the 2017 IEEE International Conference on Acoustics, Speech and Signal Processing (ICASSP), New Orleans, LA, USA, 5-9 March 2017; pp. 4845-4849.

19. Pugin, L.; Zitellini, R.; Roland, P. Verovio: A library for Engraving MEI Music Notation into SVG. In Proceedings of the 15th International Society for Music Information Retrieval Conference, ISMIR 2014, Taipei, Taiwan, 27-31 October 2014; Wang, H., Yang, Y., Lee, J.H., Eds., 2014; pp. 107-112.

20. Pacha, A.; Choi, K.Y.; Coüasnon, B.; Ricquebourg, Y.; Zanibbi, R.; Eidenberger, H. Handwritten music object detection: Open issues and baseline results. In Proceedings of the 2018 13th IAPR International Workshop on Document Analysis Systems (DAS), Vienna, Austria, 24-27 April 2018; pp. 163-168.

21. Ren, S.; He, K.; Girshick, R.; Sun, J. Faster r-cnn: Towards real-time object detection with region proposal networks. In Proceedings of the Advances in Neural Information Processing Systems, Montreal, QC, Canada, 7-12 December 2015; pp. 91-99.

22. Dai, J.; Li, Y.; He, K.; Sun, J. R-fcn: Object detection via region-based fully convolutional networks. In Proceedings of the Advances in Neural Information Processing Systems, Barcelona, Spain, 5-10 December 2016; pp. 379-387.

23. Hajič, J.; Pecina, P. The MUSCIMA++ dataset for handwritten optical music recognition. In Proceedings of the 2017 14th IAPR International Conference on Document Analysis and Recognition (ICDAR), Kyoto, Japan, 9-15 November 2017; Volume 1, pp. 39-46.

24. Calvo-Zaragoza, J.; Toselli, A.H.; Vidal, E. Early handwritten music recognition with hidden markov models. In Proceedings of the 2016 15th International Conference on Frontiers in Handwriting Recognition (ICFHR), Shenzhen, China, 23-26 October 2016; pp. 319-324.

25. Calvo-Zaragoza, J.; Toselli, A.H.; Vidal, E. Handwritten music recognition for mensural notation: formulation, data and baseline results. In Proceedings of the 2017 14th IAPR International Conference on Document Analysis and Recognition (ICDAR), Kyoto, Japan, 9-15 November 2017; Volume 1, pp. 1081-1086.

26. Miyao, H. Stave extraction for printed music scores using DP matching. J. Adv. Comput. Intell. Intell. Inform. 2004, 8, 208-215. [CrossRef]

27. Dalitz, C.; Droettboom, M.; Pranzas, B.; Fujinaga, I. A comparative study of staff removal algorithms. IEEE Trans. Pattern Anal. Mach. Intell. 2008, 30, 753-766. [CrossRef] [PubMed]

28. Calvo-Zaragoza, J.; Castellanos, F.; Vigliensoni, G.; Fujinaga, I. Deep neural networks for document processing of music score images. Appl. Sci. 2018, 8, 654. [CrossRef]

29. Wick, C.; Puppe, F. Fully Convolutional Neural Networks for Page Segmentation of Historical Document Images. In Proceedings of the 13th IAPR International Workshop on Document Analysis Systems, DAS 2018, Vienna, Austria, 24-27 April 2018; pp. 287-292. [CrossRef]

30. Rebelo, A.; Fujinaga, I.; Paszkiewicz, F.; Marcal, A.R.S.; Guedes, C.; Cardoso, J.S. Optical music recognition: state-of-the-art and open issues. Int. J. Multimed. Inf. Retr. 2012, 1, 173-190. [CrossRef] 
31. Ronneberger, O.; Fischer, P.; Brox, T. U-Net: Convolutional Networks for Biomedical Image Segmentation. In Proceedings of the MICCAI 2015-18th International Conference on Medical Image Computing and Computer-Assisted Intervention, Munich, Germany, 5-9 October 2015; Navab, N., Hornegger, J., Wells, W.M., Frangi, A.F., Eds.; Lecture Notes in Computer Science; Springer: Cham, Switzerland, 2015; Volume 9351, pp. 234-241.

32. Fujinaga, I. Staff detection and removal. In Visual Perception of Music Notation: On-Line and Off Line Recognition; IGI Global: Hershey, PA, USA, 2004; pp. 1-39.

33. Cardoso, J.S.; Rebelo, A. Robust Staffline Thickness and Distance Estimation in Binary and Gray-Level Music Scores. In Proceedings of the 20th International Conference on Pattern Recognition, ICPR 2010, Istanbul, Turkey, 23-26 August 2010; pp. 1856-1859. [CrossRef]

(C) 2019 by the authors. Licensee MDPI, Basel, Switzerland. This article is an open access article distributed under the terms and conditions of the Creative Commons Attribution (CC BY) license (http:/ / creativecommons.org/licenses/by/4.0/). 